\title{
Overview of LHCb-RICH upgrade
}

\section{LHCb-RICH collaboration}

RICH-2016, Bled, Slovenia

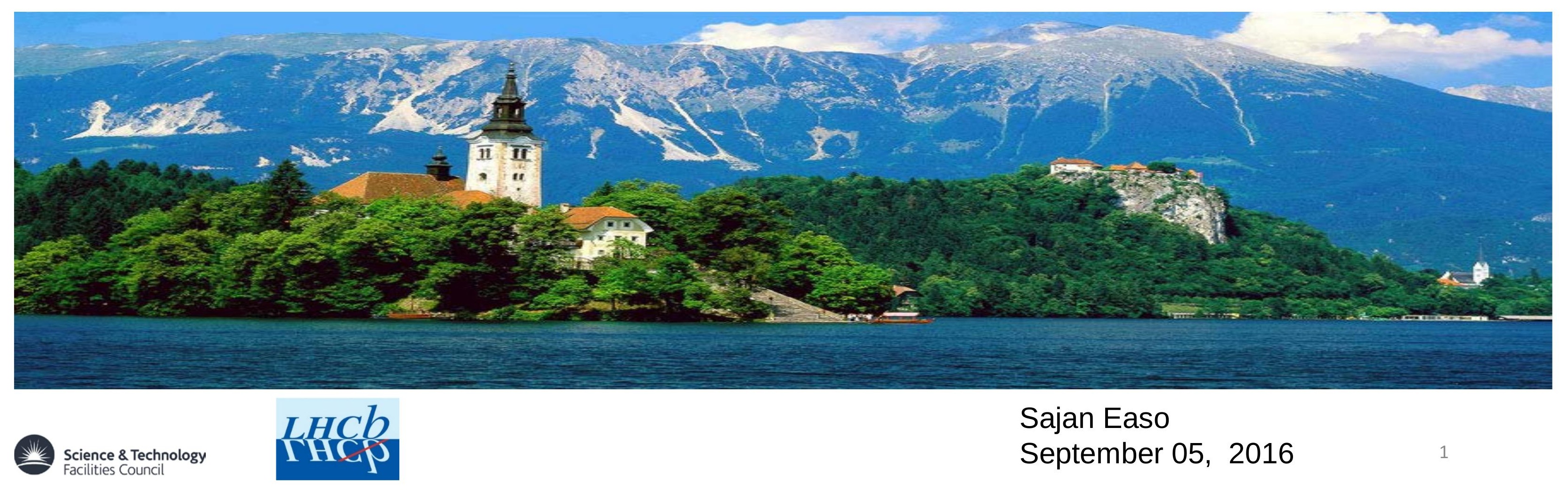




\section{The LHCb Experiment}

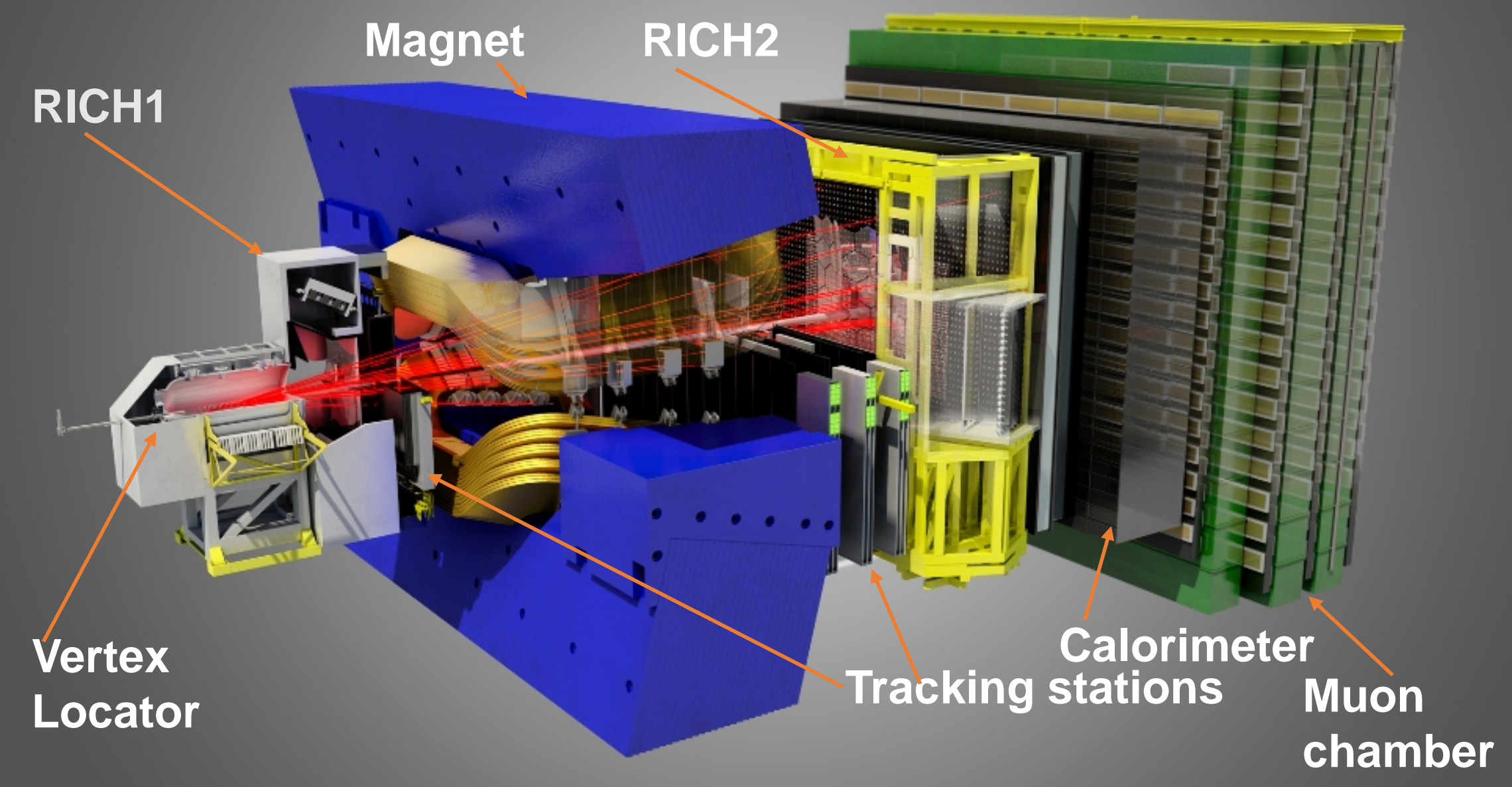

At present:

$\mathrm{pp}: \sqrt{\mathrm{s}}=13 \mathrm{TeV}$

$\mathrm{RICH} 1:$

$\mathrm{C}_{4} \mathrm{~F}_{10}<\sim 60 \mathrm{GeV} / \mathrm{c}$ Photodetectors: Top + Bottom

$\mathrm{RICH} 2:$

$\mathrm{CF}_{4}$ : > $20 \mathrm{GeV} / \mathrm{C}$ Photodetectors: Left+ Right

RICH detectors in current $\mathrm{LHCb}$ : Talk by A. Papanestis today

$2<\eta<5$

Overall acceptance $\sim 10 \rightarrow 300 \mathrm{mrad}, \quad$ Momentum range : $2-100 \mathrm{GeV} / \mathrm{c}$ 


\section{LHCb upgrade : concept}

- Search for signals of new physics(NP) beyond the standard model (SM) in high energy physics

- Indirect search from the decays of hadrons with $b$ and $c$ quarks

- Large sources of flavour symmetry breaking are excluded at TeV scale, from the current measurements

- Aim for a significant increase in the precision of LHCb measurements by increasing the readout rate in Run3
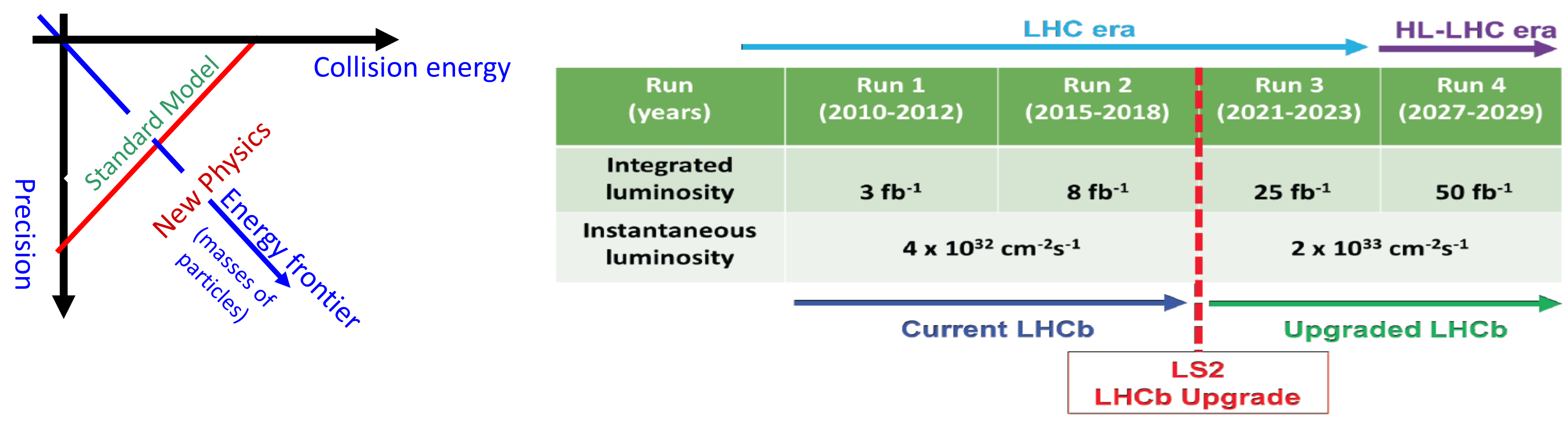

- Current upgrade to be installed during 2019-20

- Ideas for a future RICH upgrade to be installed during 2024-26: Talk by C. D'Ambrosio tomorrow 


\section{LHCb upgrade : concept}

- Main bottlenecks for going to high readout rate:

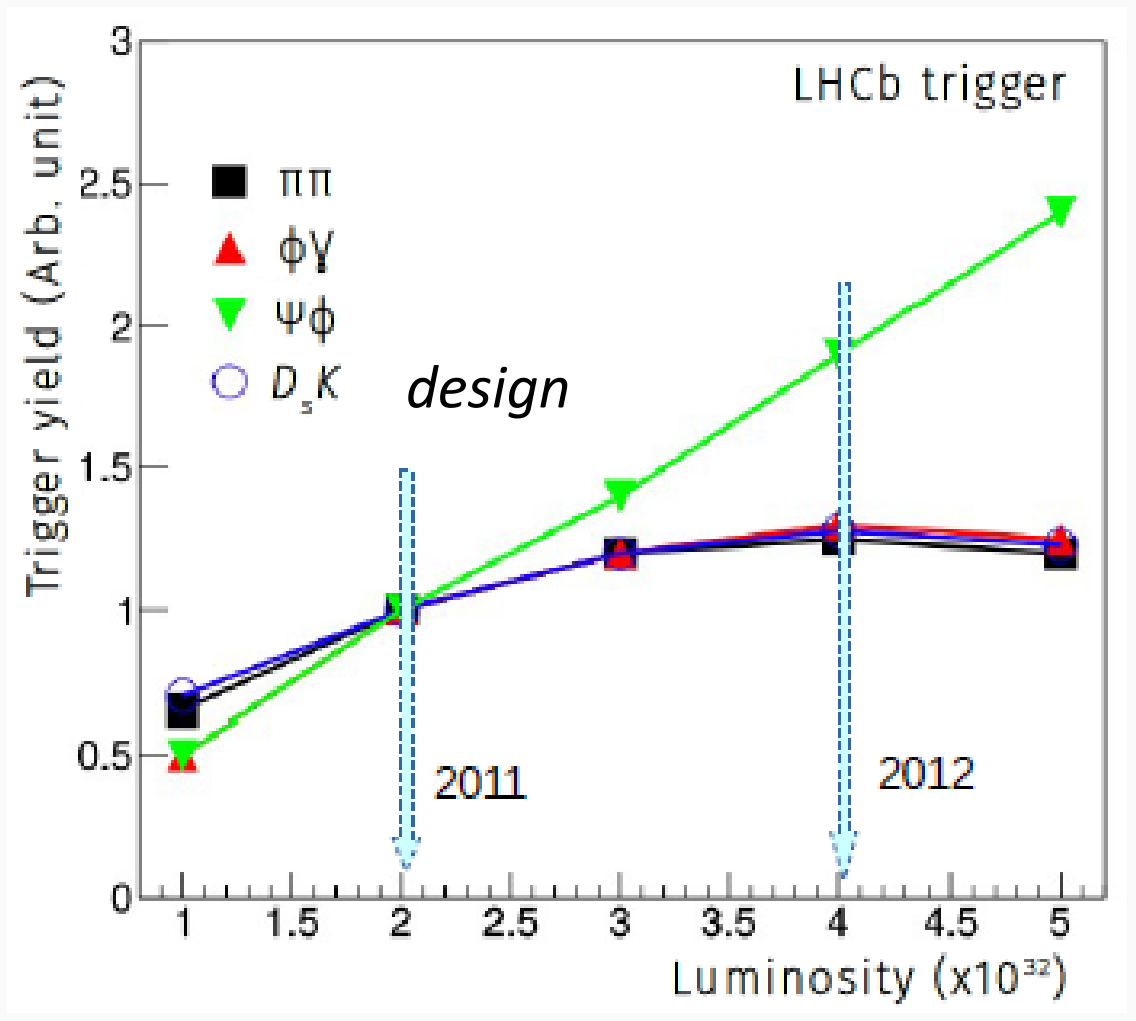

\begin{tabular}{|l|c|c|c|}
\hline Year & 2012 & 2015 & 2021 \\
\hline $\begin{array}{l}\text { Data to storage } \\
\text { in } \mathrm{kHz}\end{array}$ & 5 & 12.5 & $20-100$ \\
\hline
\end{tabular}

- Current 'Front End' max readout rate $=1.1 \mathrm{MHz}$

- Level0 hardware trigger $\left(E_{T} \& P_{T}\right.$ in calorimeter \& muon detectors): Limited discrimination power $\rightarrow$ Yields saturate in hadronic channels

LHCb 2015 Trigger Diagram

$40 \mathrm{MHz}$ bunch crossing rate

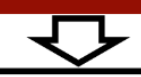

LO Hardware Trigger : 1 MHz readout, high $\mathrm{E}_{\mathrm{T}} / \mathrm{P}_{\mathrm{T}}$ signatures

\begin{tabular}{|c|c|c|}
\hline $\begin{array}{c}450 \mathrm{kHz} \\
h^{ \pm}\end{array}$ & $\begin{array}{c}400 \mathrm{kHz} \\
\mu / \mu \mu\end{array}$ & $\begin{array}{c}150 \mathrm{kHz} \\
\mathrm{e} / \mathrm{Y}\end{array}$ \\
\hline
\end{tabular}

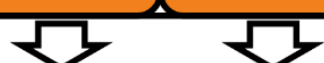
Software High Levei Trigger

Partial event reconstruction, select displaced tracks/vertices and dimuons ए人

Buffer events to disk, perform online detector calibration and alignment

Full offline-like event selection, mixture
of inclusive and exclusive triggers

LHCb Upgrade Trigger Diagram

$30 \mathrm{MHz}$ inelastic event rate (full rate event building)

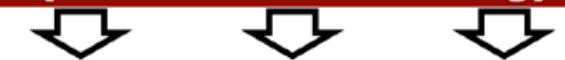
Software High Level Trigger

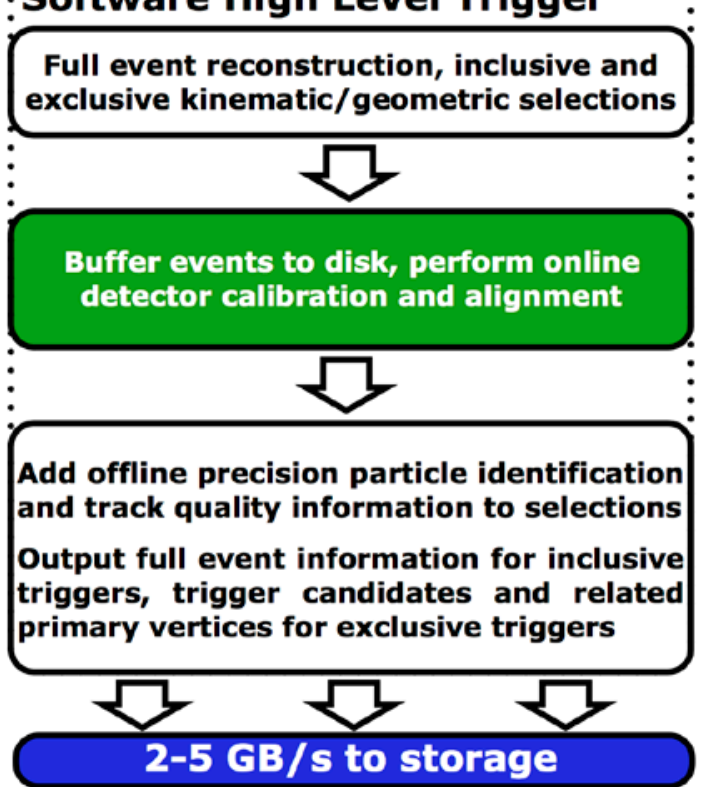

Optional low level hardware Trigger (LLT) with low thresholds 


\section{$\mathrm{RICH}$ in the $\mathrm{LHCb}$ upgrade}

- Photodetectors:

- Replace HPDs : Pixel chip encapsulated in each HPD has $1 \mathrm{MHz}$ max. readout rate

- Use new MaPMTs from Hamamatsu : each with 8 X 8 pixels

$>\mathrm{R} 13742$ (from $\mathrm{R} 11265$ series) : Active area : $23 \times 23 \mathrm{~mm}^{2}$

$>$ R13743 (from H12700 series) : Active area : $48 \times 48 \mathrm{~mm}^{2}$

- R13742 for RICH1 and inner region of RICH2

- R13743 for outer regions of RICH2

- Readout :

- CLARO: custom made ASIC

- New readout chain
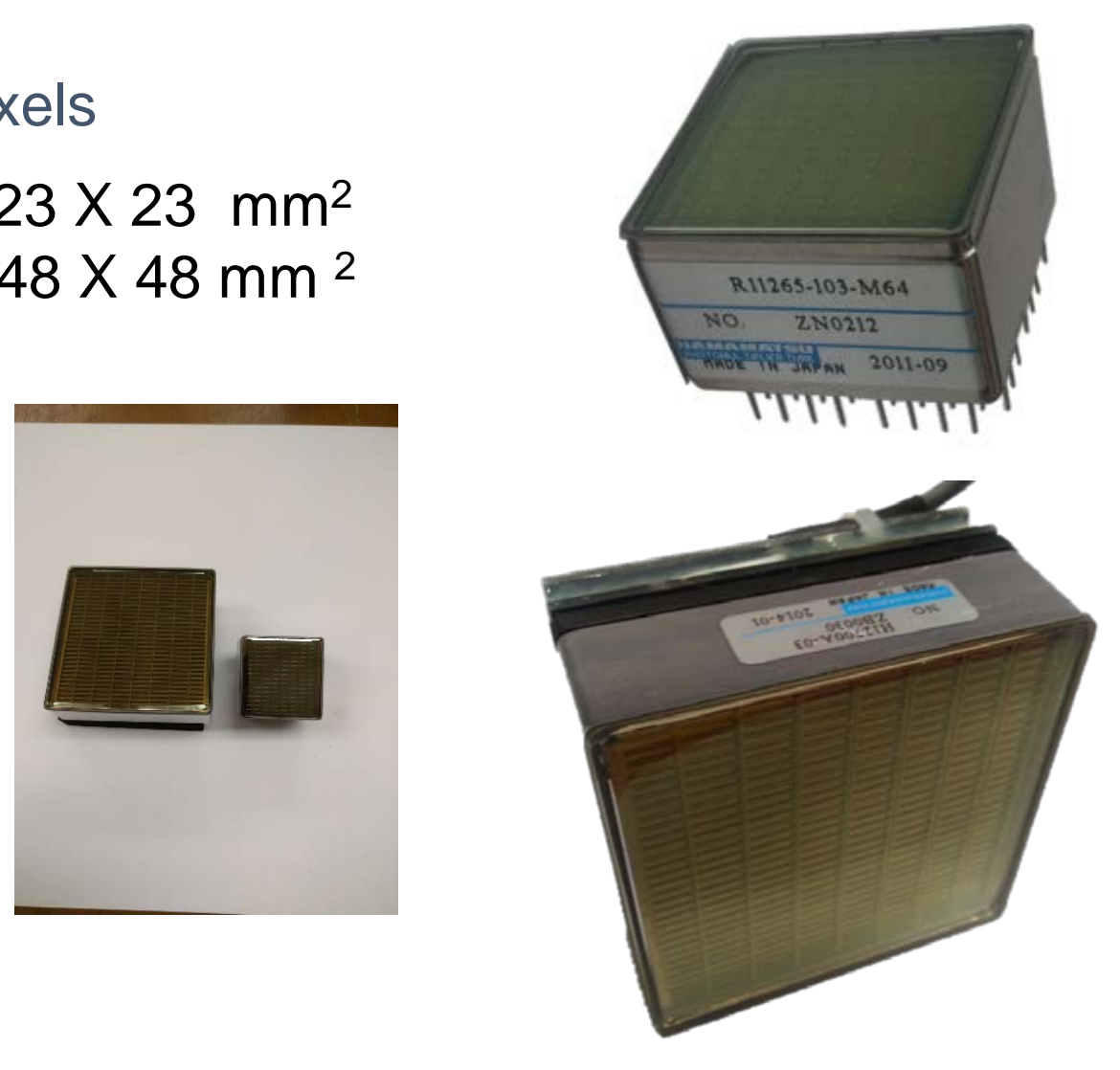


\section{$\mathrm{RICH} 1$ in $\mathrm{LHCb}$ upgrade}

- Improved optics in RICH1 to obtain optimal PID performance at the increased luminosity

- The upgraded RICH1 has similar structure to that of the current RICH1, but it is completely revamped

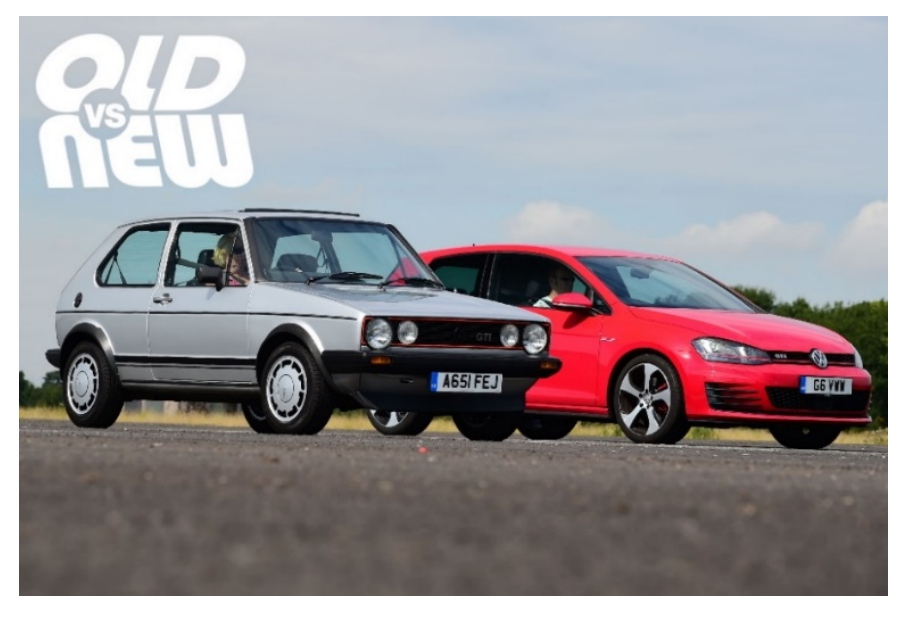

WW Golf GTI : Mk I vs Mk VII

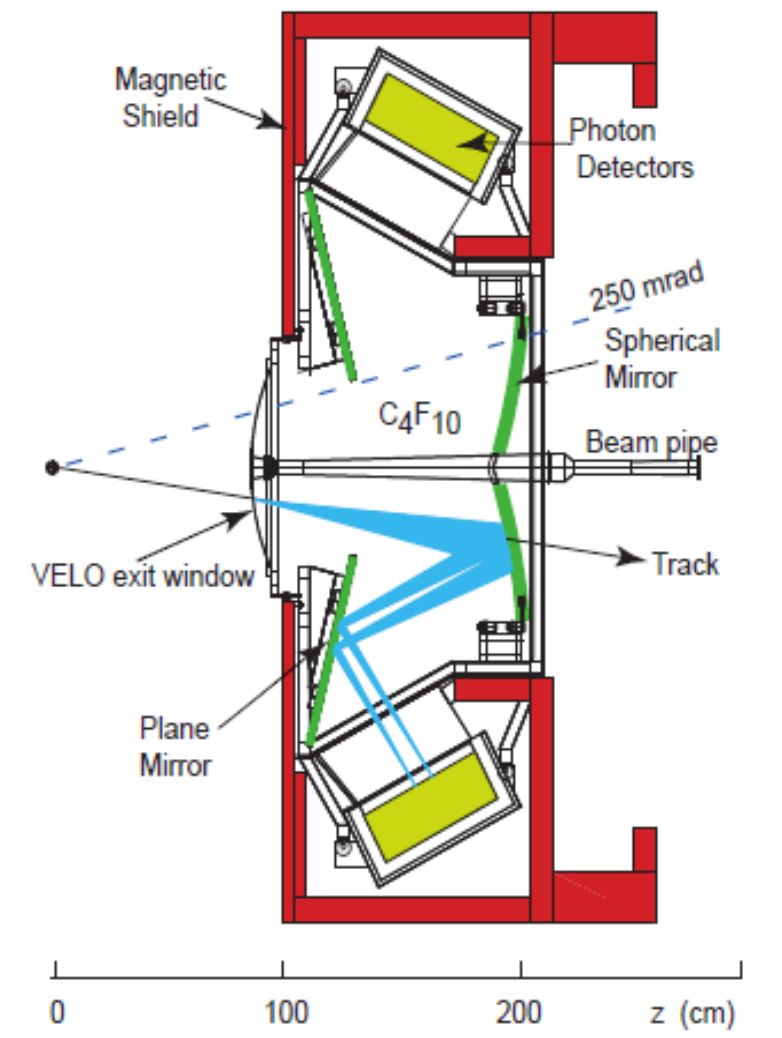

- Spherical mirror ROC: $2710 \rightarrow 3650 \mathrm{~mm}$ to reduce the occupancy at high luminosity

- New mechanical structure to the install the arrays of MaPMTs and their readout boards.

- New mirrors, new box which contains the mirrors and the radiator gas.

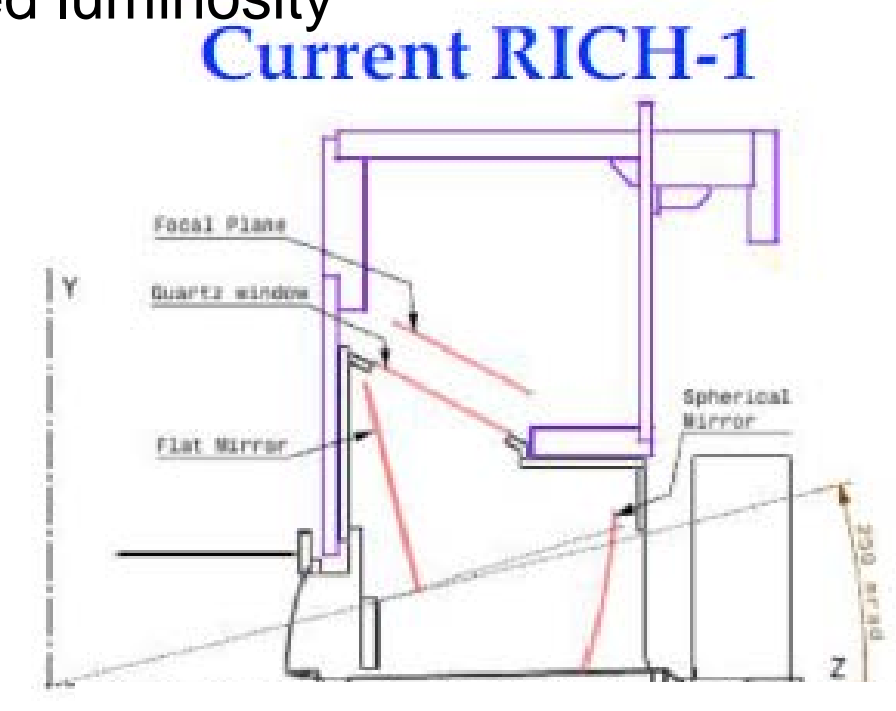

\section{Upgraded RICH-1}

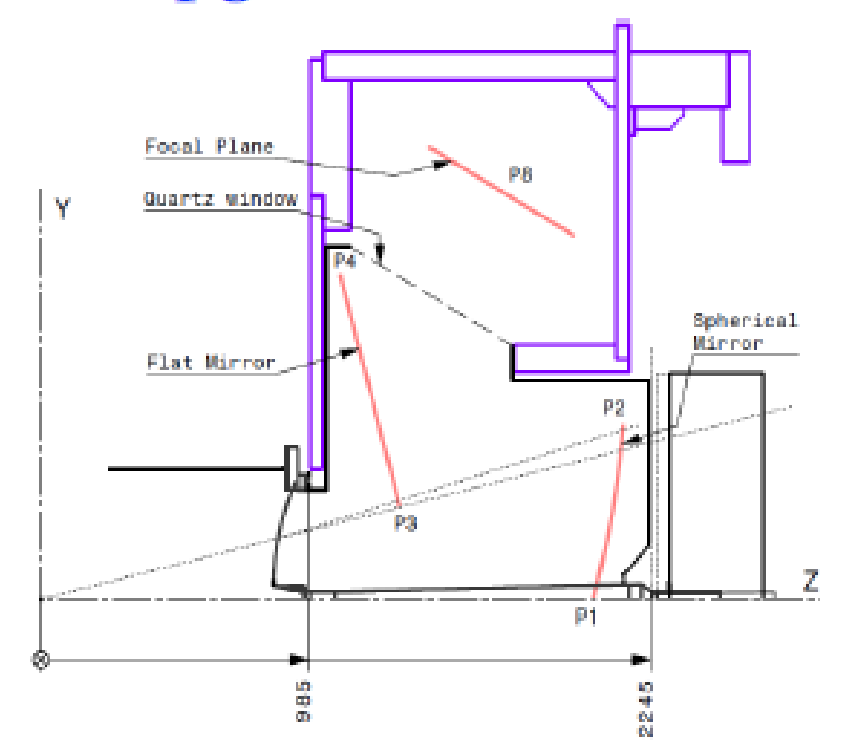



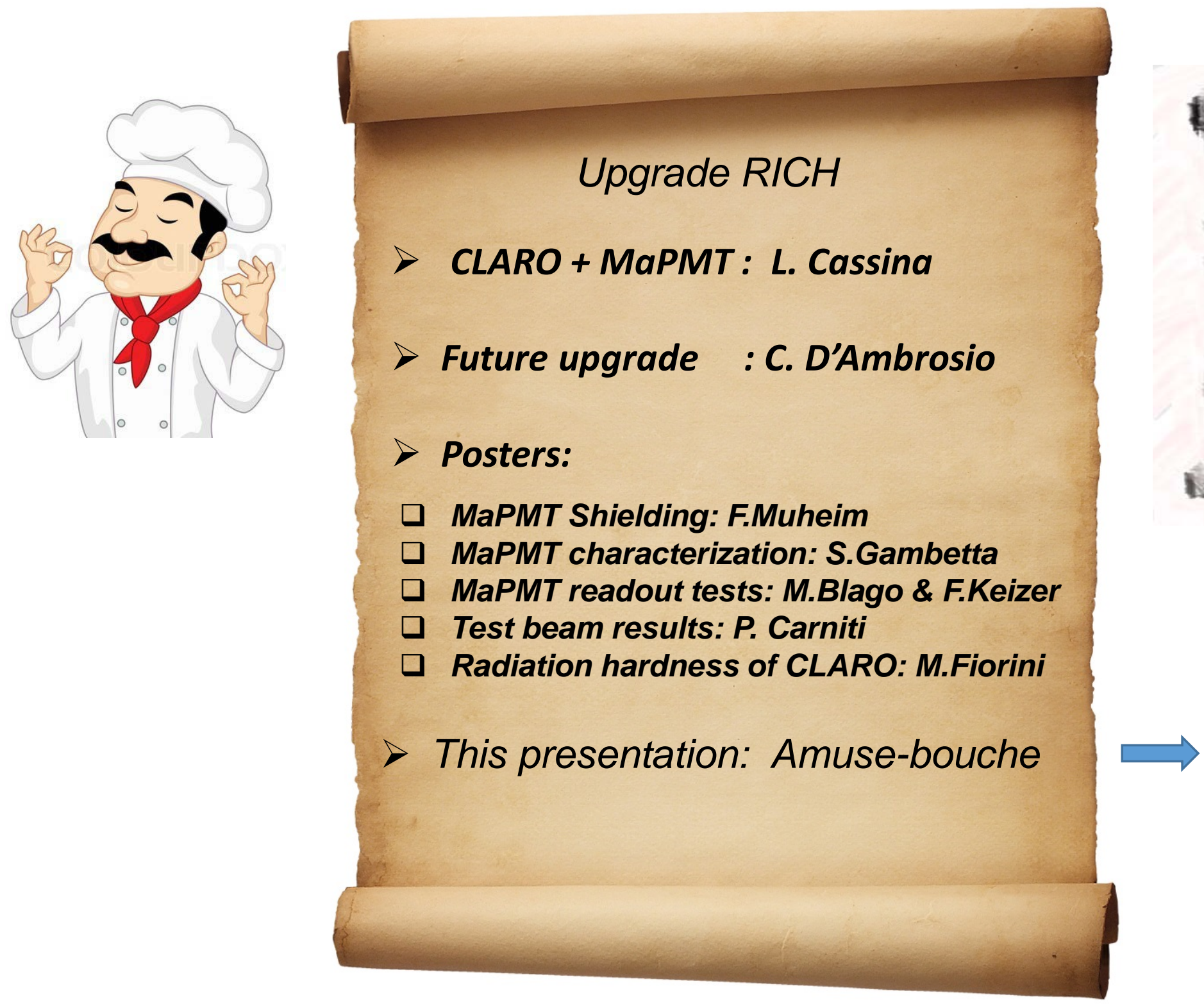

\section{Current RICH}

\section{Performance : A.Papanestis}

Real time calibration \& alignment : J.He

- Performance from simulations

* RICH1 and RICH2 mechanics development

* MaPMTs, readout

* Magnetic shielding

* Prototype testing 


\section{PID performance from simulations}

- LHCb tracking detectors are also being upgraded.

- In the simulations, the upgraded tracking system is used along with upgraded RICH

- Simulations: PYTHIA v8 + EVTGEN + GEANT4

- Reconstructed tracks and RICH hits are subjected to a log-likelihood algorithm for PID

- Results quoted in terms of the difference in the log-likelihood (DLL) between kaons and pions

\begin{tabular}{|c|c|c|c|c|c|}
\hline $\begin{array}{l}\text { Configuration } \\
\text { for simulation }\end{array}$ & $\begin{array}{l}\text { Luminosity } \\
10^{32} \mathrm{~cm}^{-2} \mathrm{~s}^{-1}\end{array}$ & $\begin{array}{l}\text { nominal } \\
\text { \# of } \\
\text { Bunches }\end{array}$ & $\begin{array}{l}\text { E } \\
\text { TeV }\end{array}$ & $\mathbf{v}$ & $v=$ Average number of pp interactions per \\
\hline Current LHCb in Run 2 & 4.0 & 2300 & 13 & 1.6 & \\
\hline Upgrade LHCb in Run 3 & 20 & 2400 & 14 & 7.6 & \\
\hline
\end{tabular}




\section{Resolutions and yields}

Single photon resolutions from full simulations:

\begin{tabular}{|c|c|c|c|c|}
\hline $\begin{array}{l}\text { Resolution } \\
\text { (in mrad) }\end{array}$ & $\begin{array}{l}\text { RICH1-2015 } \\
\text { current HPD }\end{array}$ & $\begin{array}{l}\text { RICH1-upgrade } \\
\text { MaPMT }\end{array}$ & $\begin{array}{l}\text { RICH2-2015 } \\
\text { current HPD }\end{array}$ & $\begin{array}{l}\text { RICH2-upgrade } \\
\text { MaPMT }\end{array}$ \\
\hline Chromatic & 0.84 & 0.58 & 0.48 & 0.31 \\
\hline Pixel & $\begin{array}{l}0.60 \\
P S F=0.86\end{array}$ & 0.44 & $\begin{array}{l}0.19 \\
P S F=0.29\end{array}$ & 0.19 \\
\hline Emission point & 0.76 & 0.37 & 0.27 & 0.27 \\
\hline $\begin{array}{l}\text { Overall } \\
\text { Overall+Track }\end{array}$ & $\begin{array}{l}1.60 \\
1.65\end{array}$ & $\begin{array}{l}0.78 \\
0.88\end{array}$ & $\begin{array}{l}0.65 \\
0.76\end{array}$ & $\begin{array}{l}0.45 \\
0.60\end{array}$ \\
\hline Yield & 32 & 42 & 24 & 22 \\
\hline
\end{tabular}

Using RICH2 MaPMTS in central region

Resolutions improved for upgraded geometry

Improvements in $\left\{\begin{array}{l|l}\text { Chromatic error } & \begin{array}{l}\text { MaPMT QE peaked at higher } \\ \text { wavelength than that of HPD }\end{array} \\ \hline \text { RICH1: Emission point error } & \text { Improved optics } \\ \text { RICH1: Yield } & \text { Increased radiator length }\end{array}\right.$




\section{Occupancies in $\mathrm{RICH} 1$ and $\mathrm{RICH} 2$ for upgrade}

\section{$X Y$ coordinates of hits on detector plane}

\section{$\mathrm{RICH} 1$}

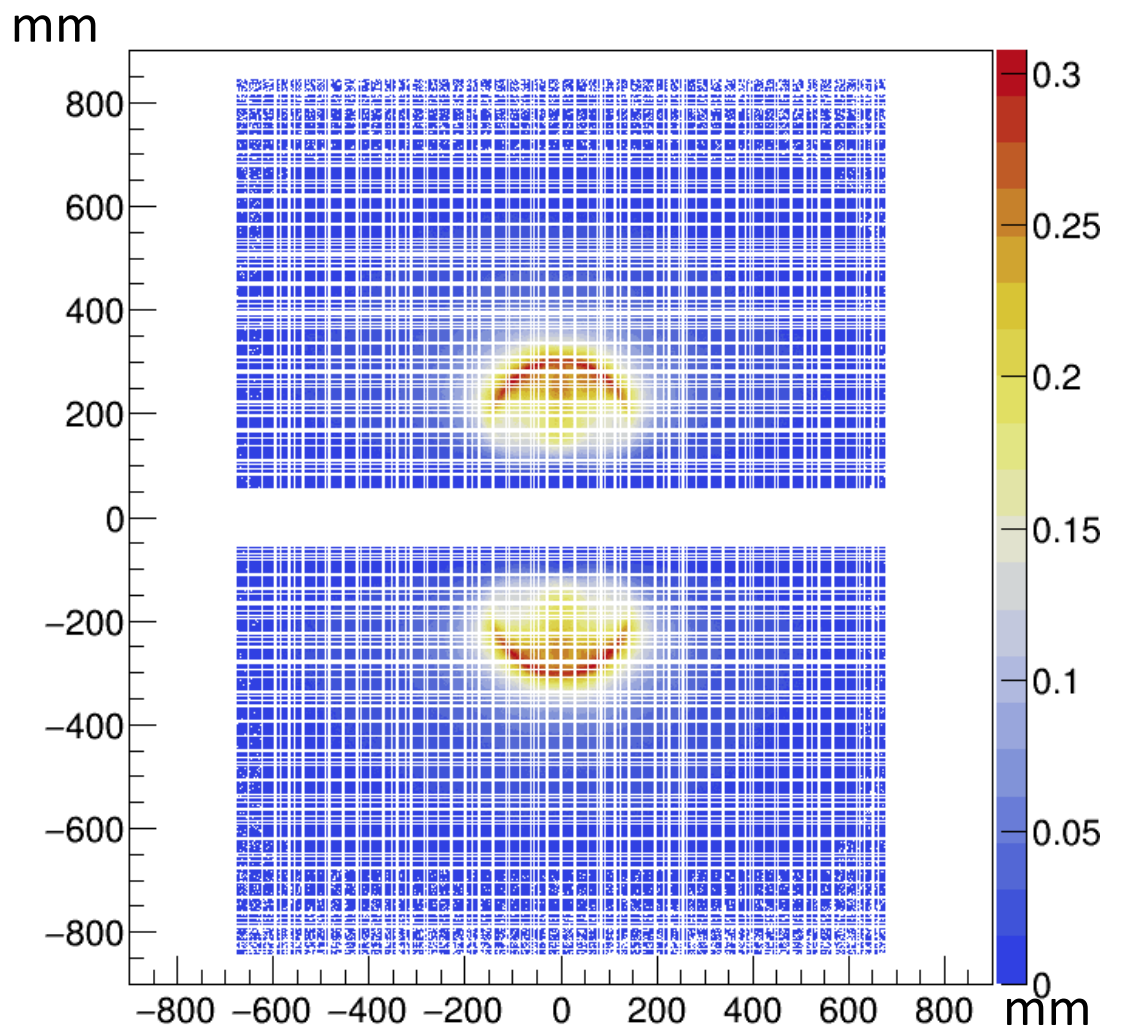

\section{$\mathrm{RICH} 2$}

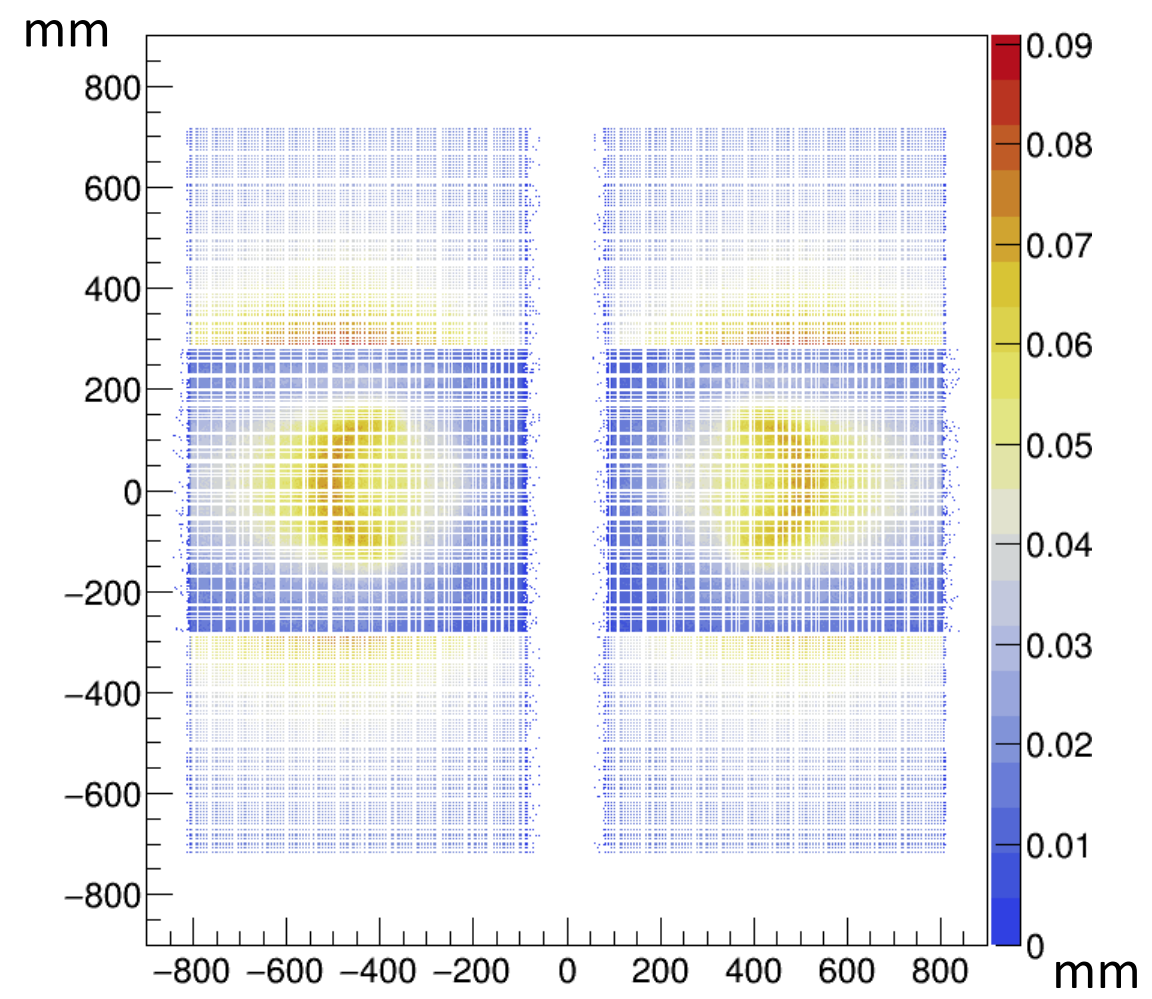

- Using upgrade geometry configuration with Run 3 luminosity

- Peak occupancy in RICH1: - $27 \%$
- MaPMT R13742 : In RICH1 and inner region of RICH2

- MaPMT R13743 : In outer region of RICH2

- If 2015 geometry configuration were used: 


\section{PID performance}

Red: Kaon identification efficiency

Blue: Probability for pion to be misidentified as kaon

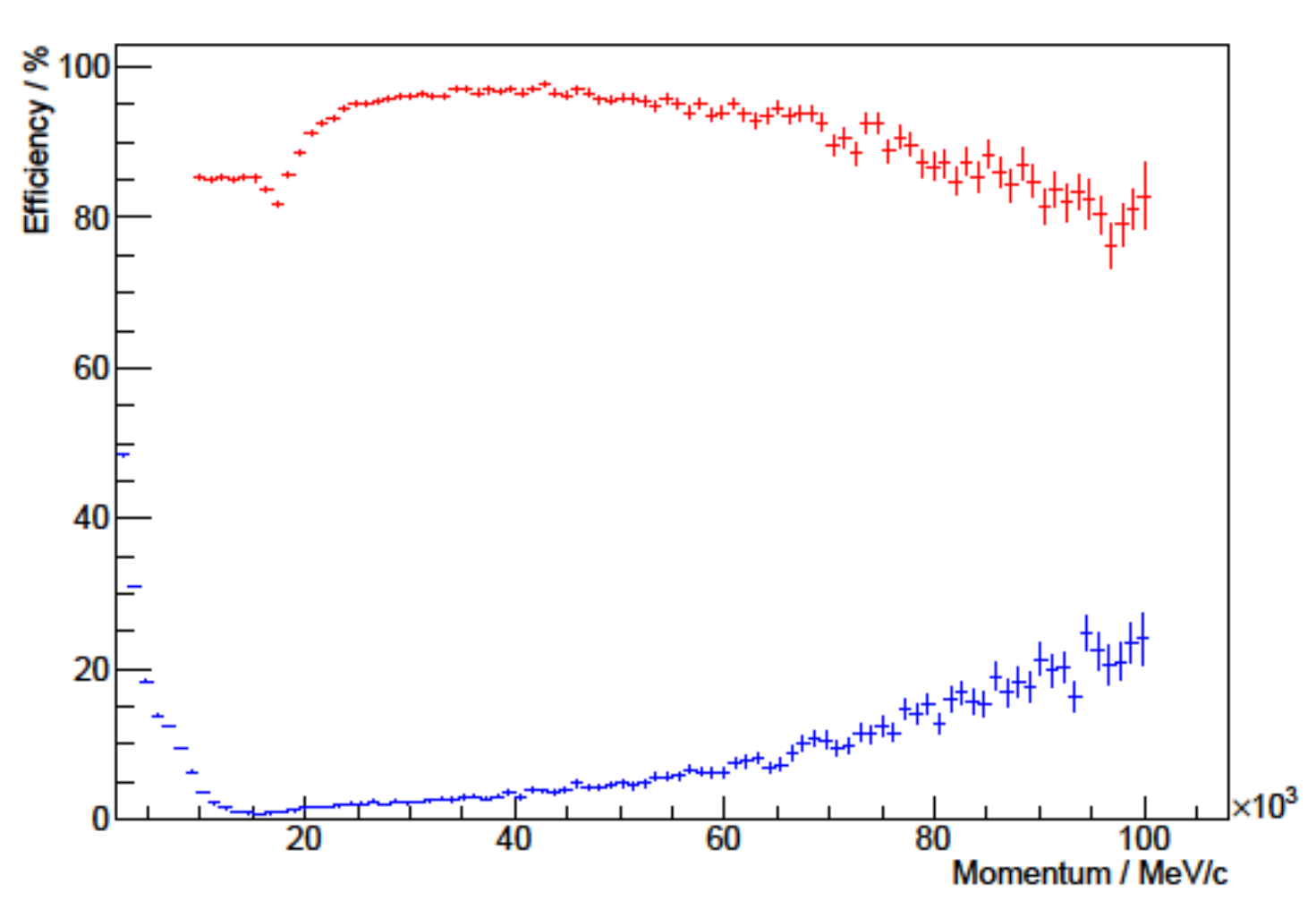

PID performance vs momentum at upgrade luminosity
RICH Kaon ID

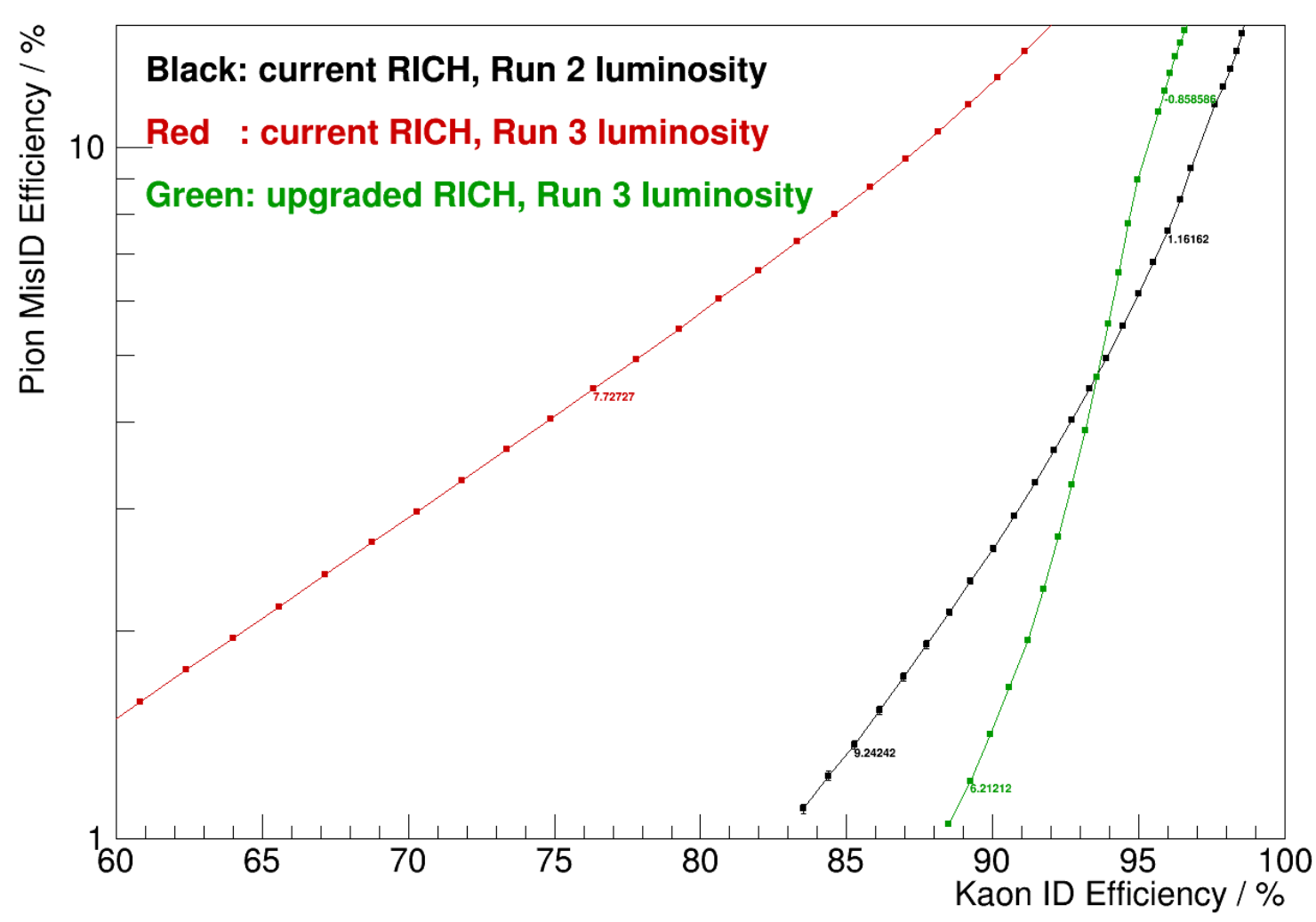

Average PID in the momentum range 2-100 GeV/c for different DLL cuts

Improved performance obtained for upgrade configuration 


\section{$\mathrm{RICH}$ components at $\mathrm{LHCb}$ upgrade}

Radiators: - Keep the same as those for the current system

- $\mathrm{C}_{4} \mathrm{~F}_{10}$ for $\mathrm{RICH} 1$ and $\mathrm{CF}_{4}$ for $\mathrm{RICH} 2$

- At low momentum, use veto mode (as a threshold counter)

- examples: below $9.3 \mathrm{GeV} / \mathrm{c}$ for $\pi / \mathrm{K}$, below $17.7 \mathrm{GeV} / \mathrm{c}$ for K/p etc.

- R\&D to improve the low momentum PID in progress

- Talk on photonic crystals by I. Kaminer

- Talk on TORCH (based on TOF method) by T.Gys Today

RICH1 Mirrors:

- New spherical mirrors to be made of carbon fibre, as in the current system.

- New flat mirrors to be made of glass, as in the current system.

- Prototypes being tested to verify reflectivity, ROC etc.

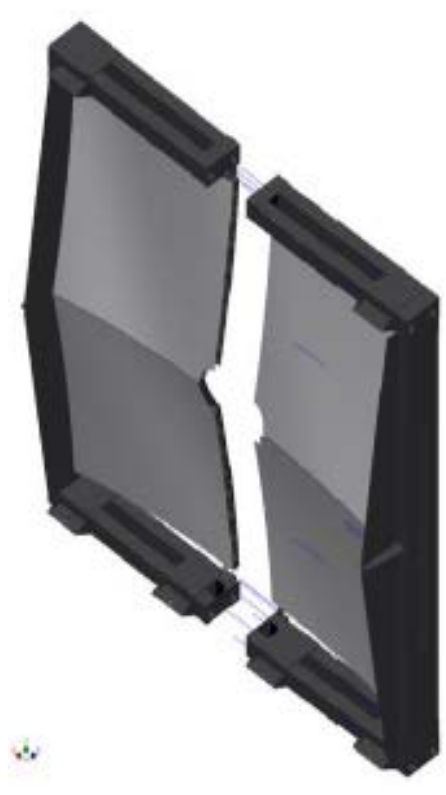



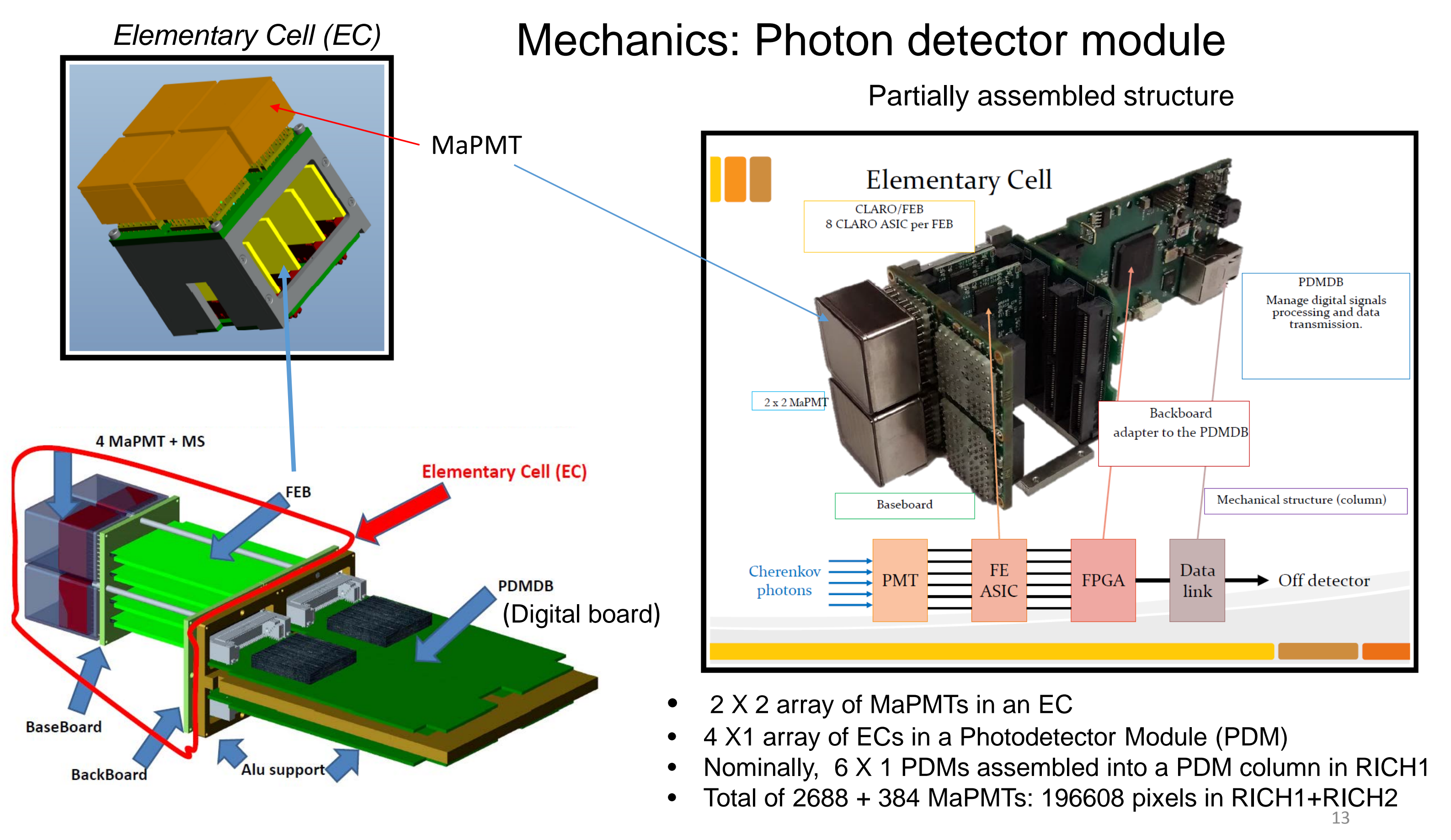


\section{RICH1 Mechanics}

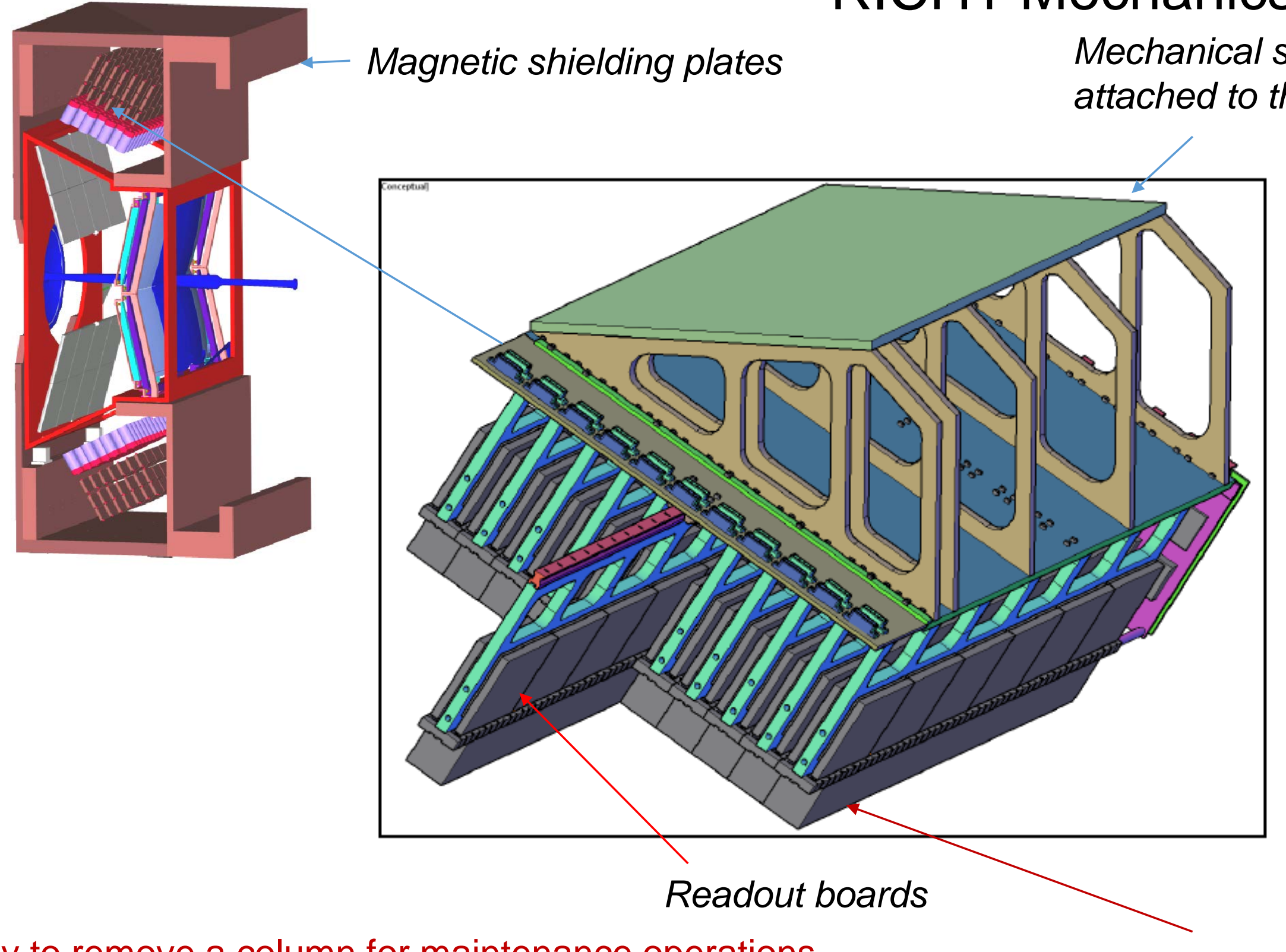

Mechanical structure attached to the shielding plates

Easy to remove a column for maintenance operations 
- Maximum magnetic field expected in the detector region of RICH1: 25 Gauss, RICH2: 5 Gauss

- This can lead to reduction of detection efficiency.

- Lab tests performed to measure detection efficiency of MaPMT prototypes for different fields

- Local shielding to be applied using a 'cross-shaped' structure made of a material similar to 'mu-metal', on the EC
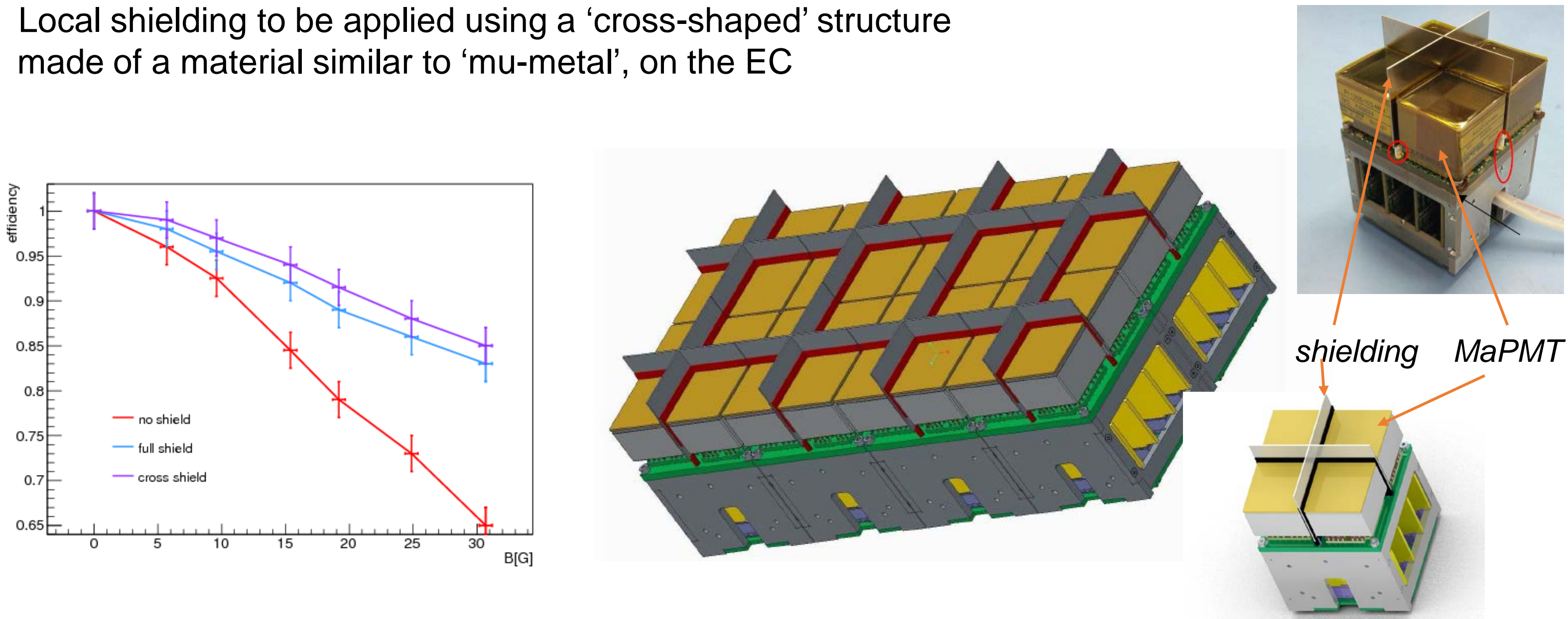


\section{RICH2 Mechanics}

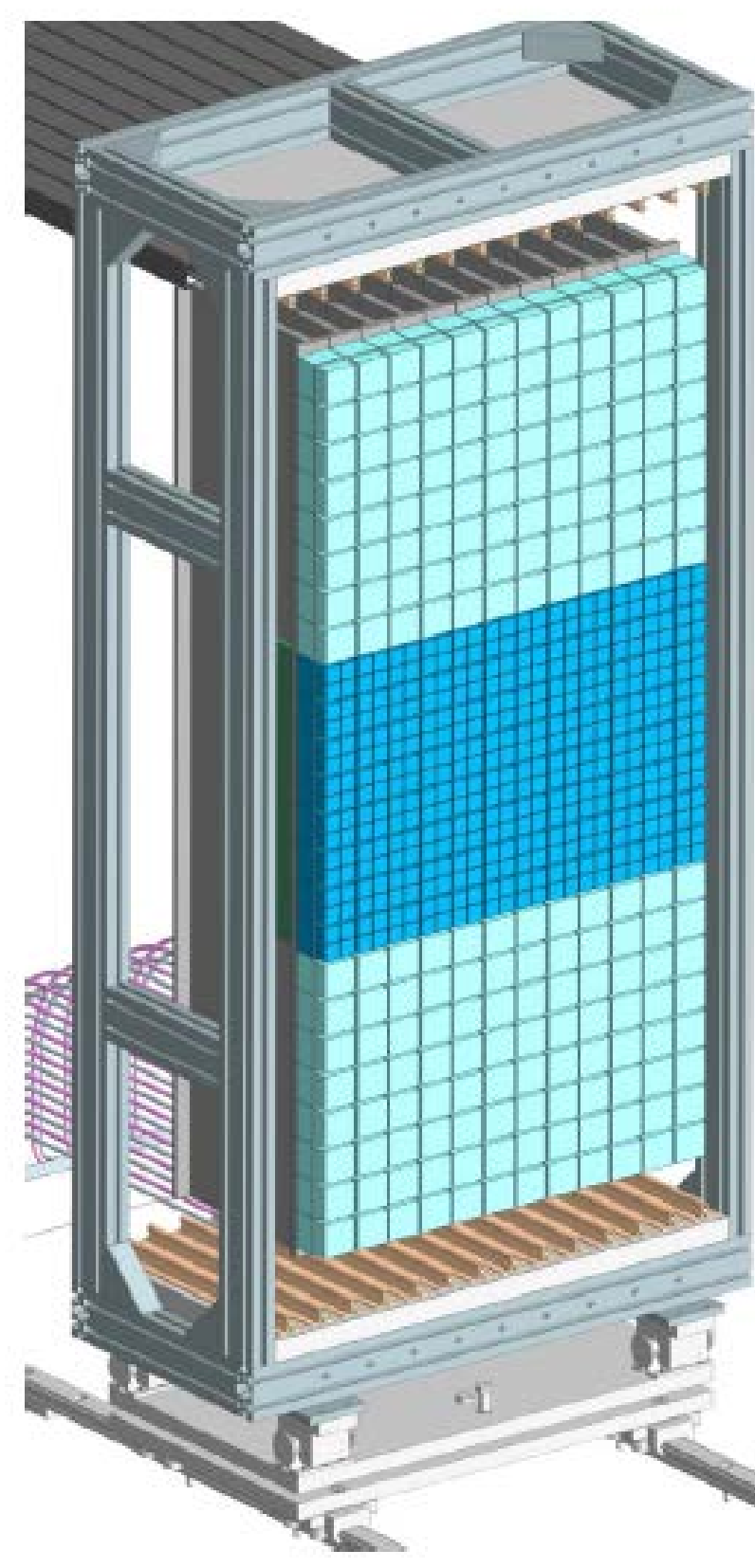

- $\mathrm{RICH} 2$ optical layout same as that in the current detector

- Two types of MaPMTs :

- Central region with R13742, same as that for RICH1

- Peripheral region R13743 with pixel size $6 \mathrm{~mm} \times 6 \mathrm{~mm}$

- Versions of EC adapted for R13743 designed and prototypes tested
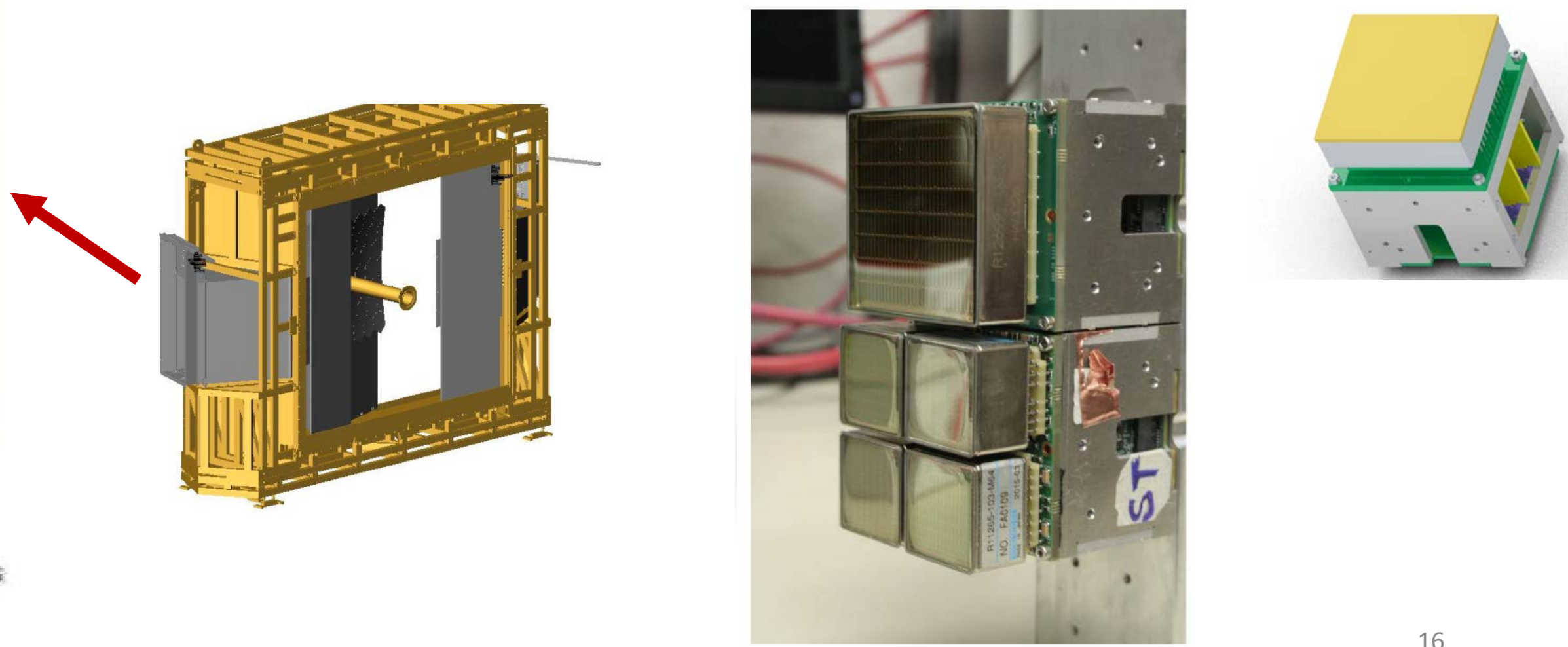


\section{$\mathrm{RICH}$ Photodetectors}

Typical QE

- Number of MaPMTS : 2700 (type R13742) + 400 (Type R13743) Pixels in each MaPMT : $8 \times 8$

- Active area fraction: R13742: $77 \% \quad(23 \mathrm{~mm} / 26.2 \mathrm{~mm})$ $\mathrm{R} 13743: 87 \% \quad(48 \mathrm{~mm} / 52 \mathrm{~mm})$

- gain $1.0 \times 10^{6}$ with a variation less than $1: 4$ and 1:3

- Average cross-talk : $<5 \%$

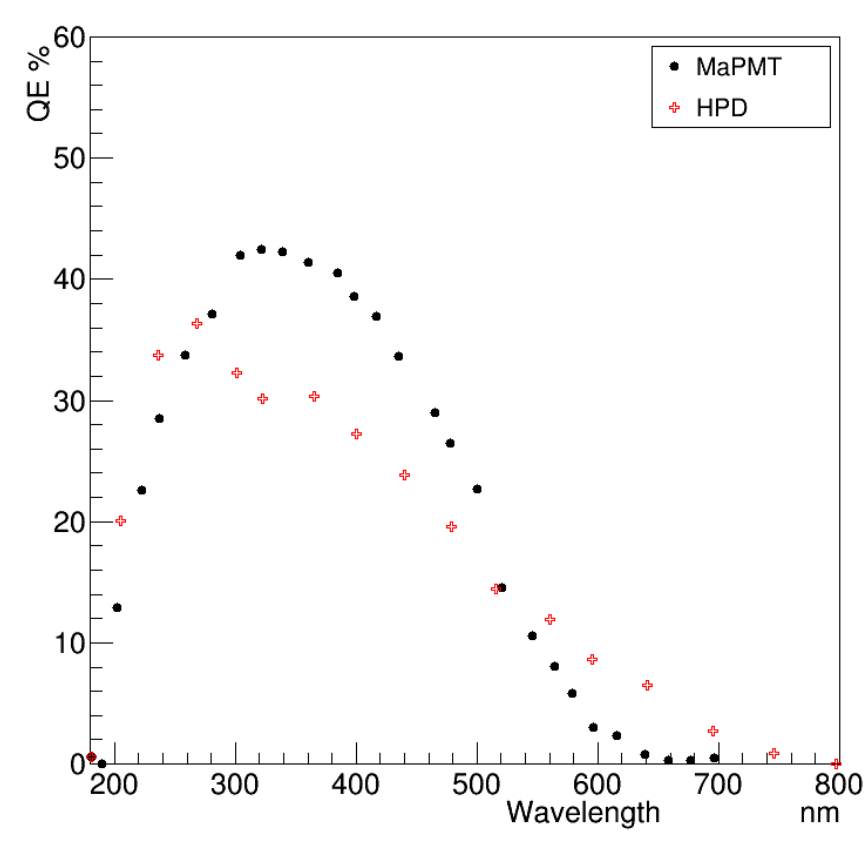

- Quality verification of each of these MaPMTs to be done in LHCb-RICH test centres
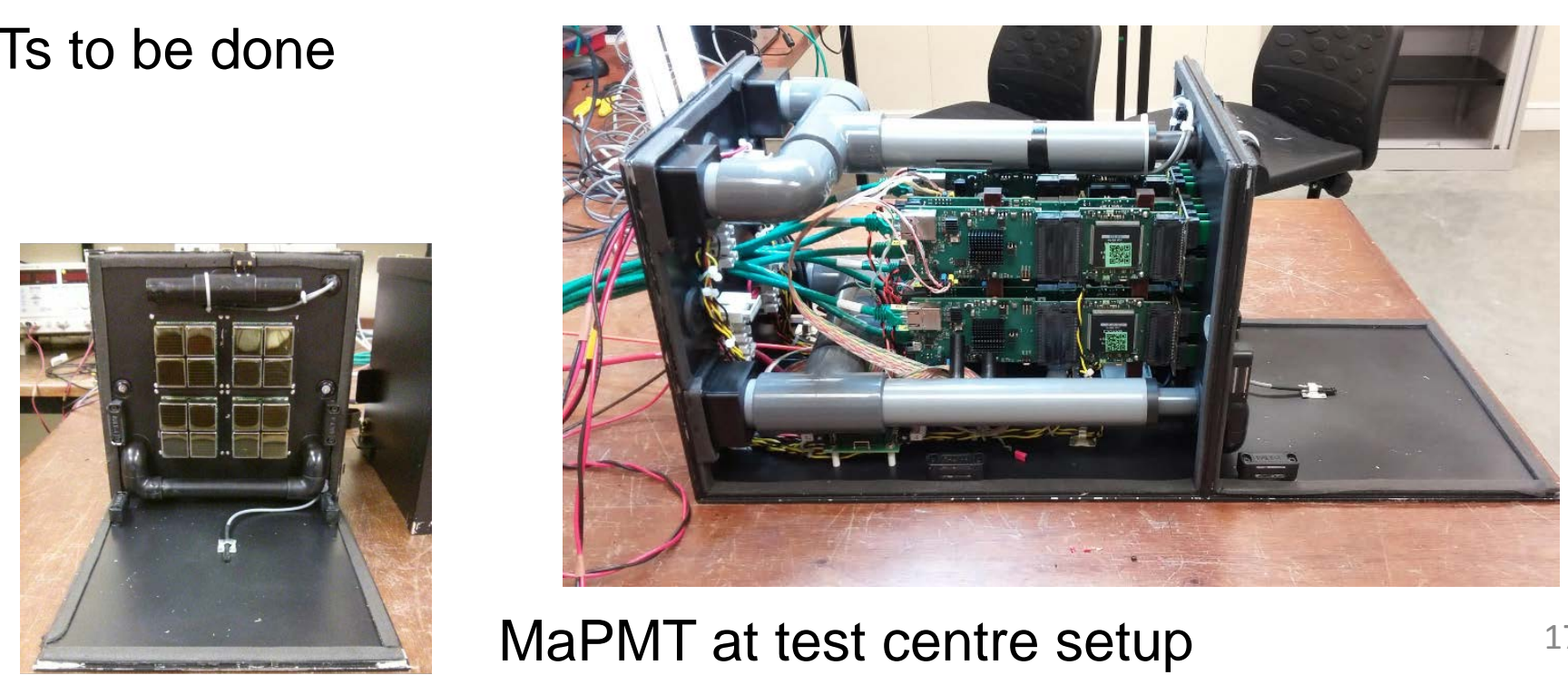


\section{RICH Readout}

\section{CLARO : 8 channel ASIC}

- 64 possible threshold settings from $30 \mathrm{ke}^{-}$to $2 \mathrm{M} \mathrm{e}^{-}$

- 3 attenuation levels

- Power consumption only $1 \mathrm{~mW}$ per channel

- Recovery time less than 25 ns.

- Radiation tolerant up to $\sim 1$ Mrad (10 k Gy) TID (Even better results obtained from recent tests)

- Performances of prototypes verified using lab tests and beam tests
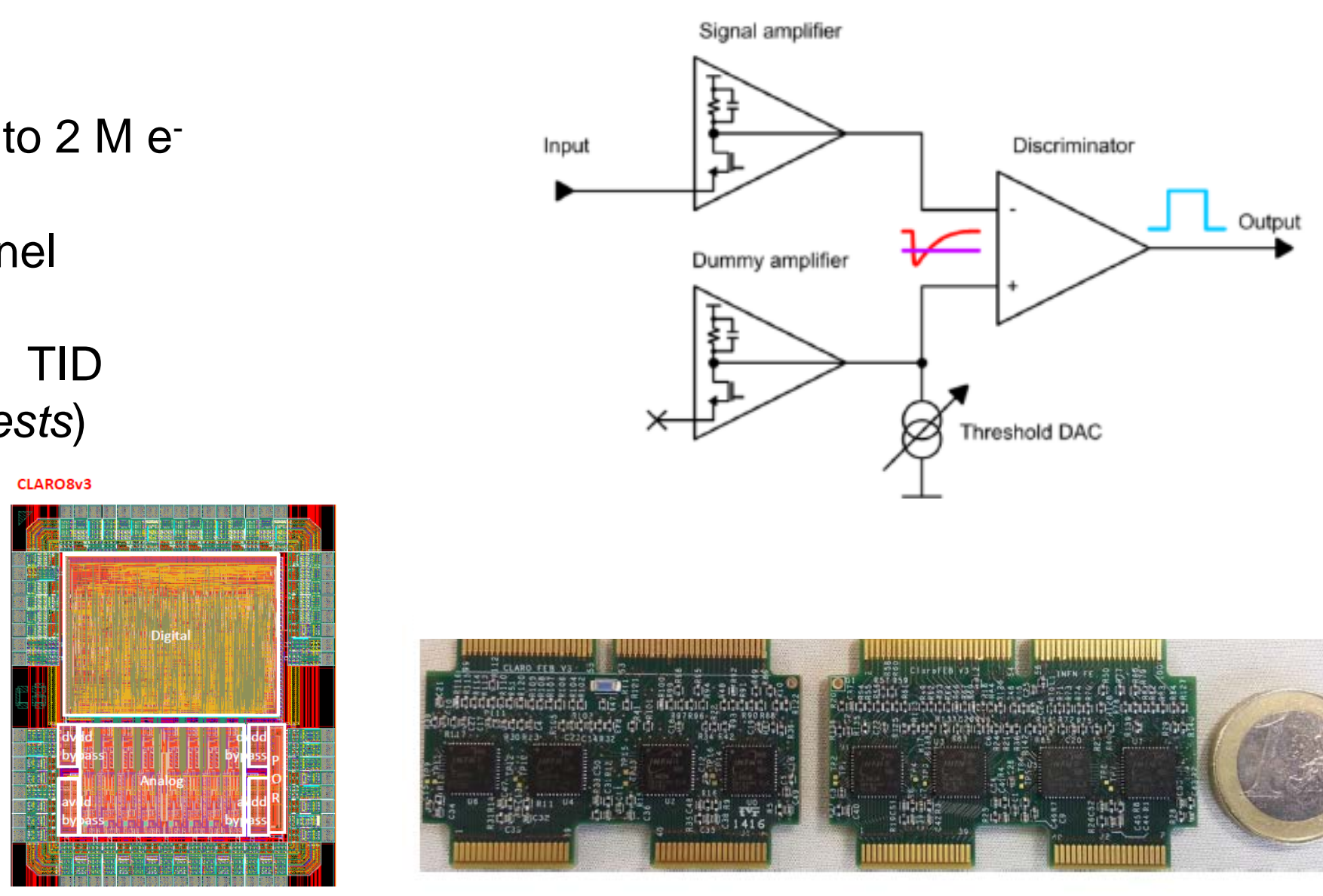

- Production of the CLARO chips expected to start soon. LHCb-RICH needs about 28000 chips.

- Characterization will be done in the test centres

Poster from M.Blago \& F.Keizer 


\section{Prototype testing}

- $180 \mathrm{GeV} / \mathrm{c}$ pions incident on a spherical segment of quartz

- The detector plane equipped with arrays of prototypes of MaPMTs, CLARO, digital readout boards and EC.

- Several test beam runs in the last three years to test various versions of these.

Number of hits per track in test beam

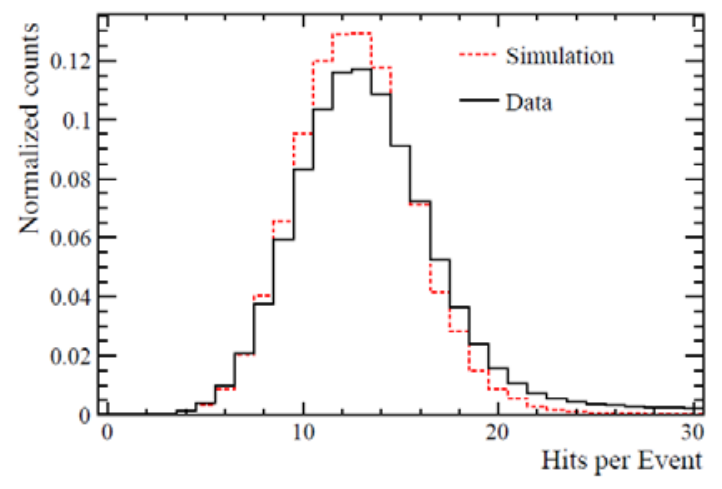

Overall resolution per photon

\begin{tabular}{|l|l|}
\hline Simulation & $17.0 \pm 1.3 \mathrm{mrad}$ \\
\hline Real data & $17.0 \pm 0.8 \mathrm{mrad}$ \\
\hline
\end{tabular}

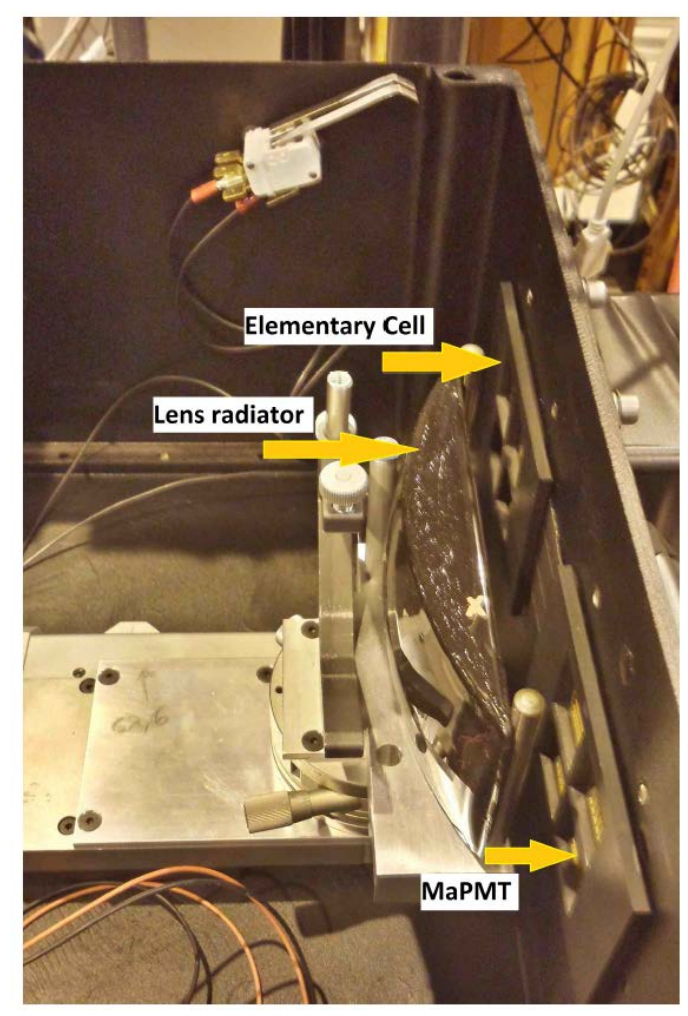

Cherenkov ring from RICH test beam in 2015
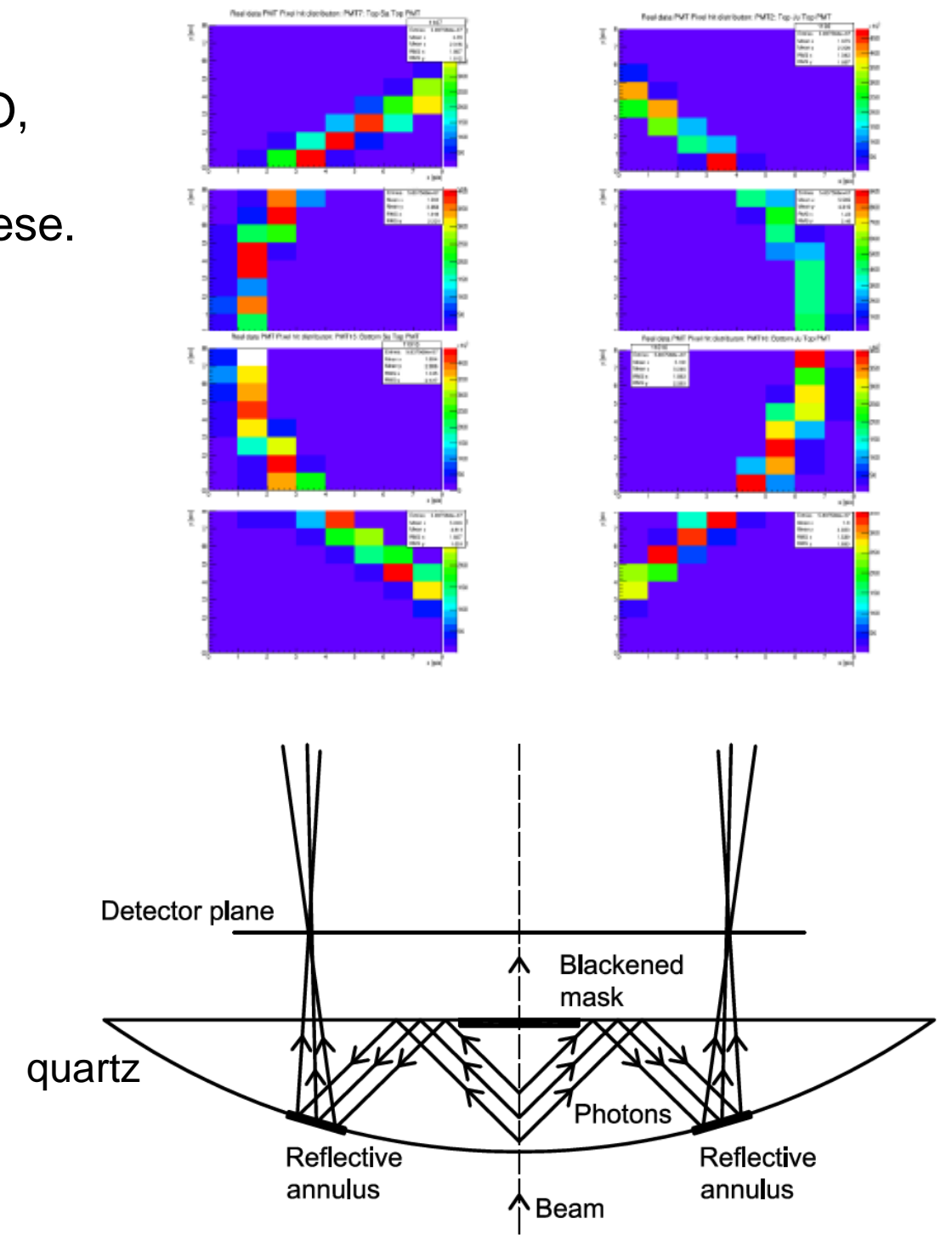


\section{Prototype testing}

Typical pulse height spectra

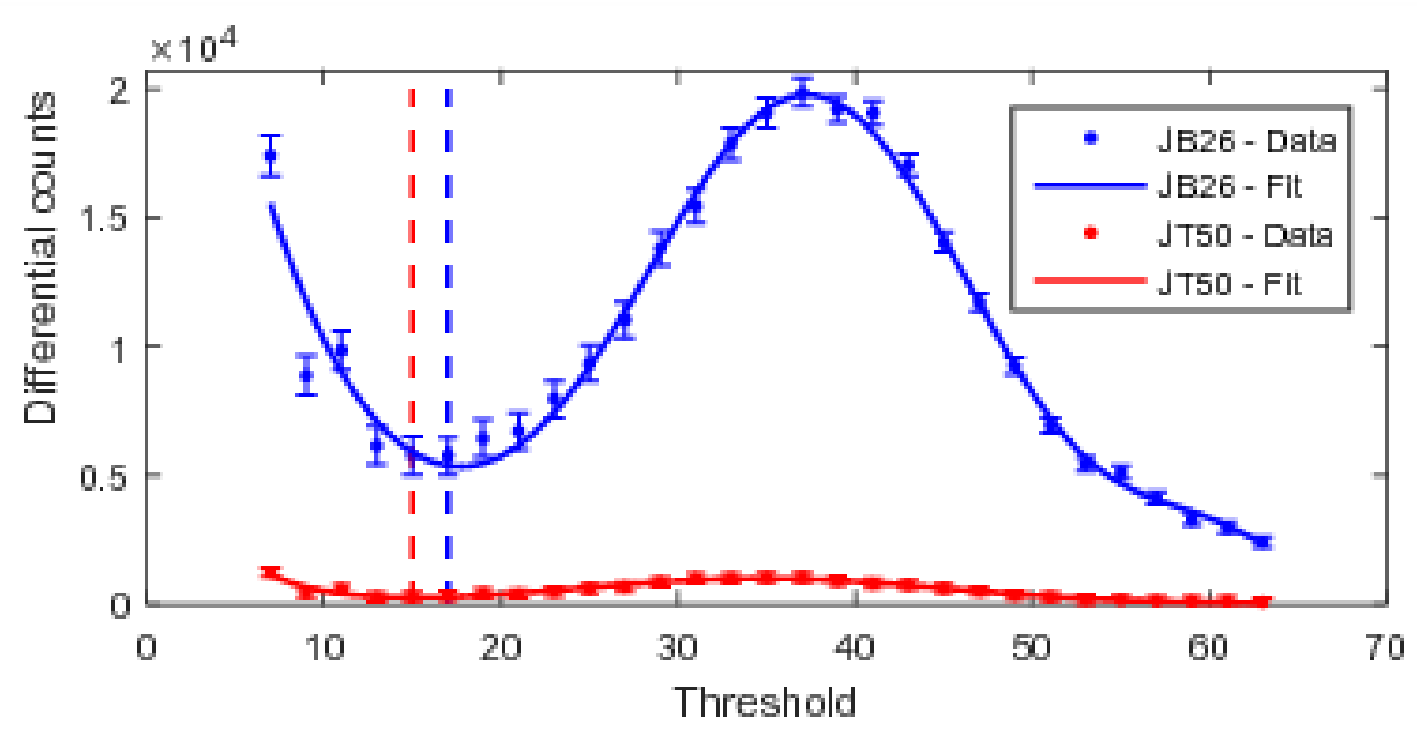

- Threshold scans:

Threshold unit $=35 \times 10^{3} \mathrm{e}$

- Collect data with different thresholds

- Determine the pulse height spectra

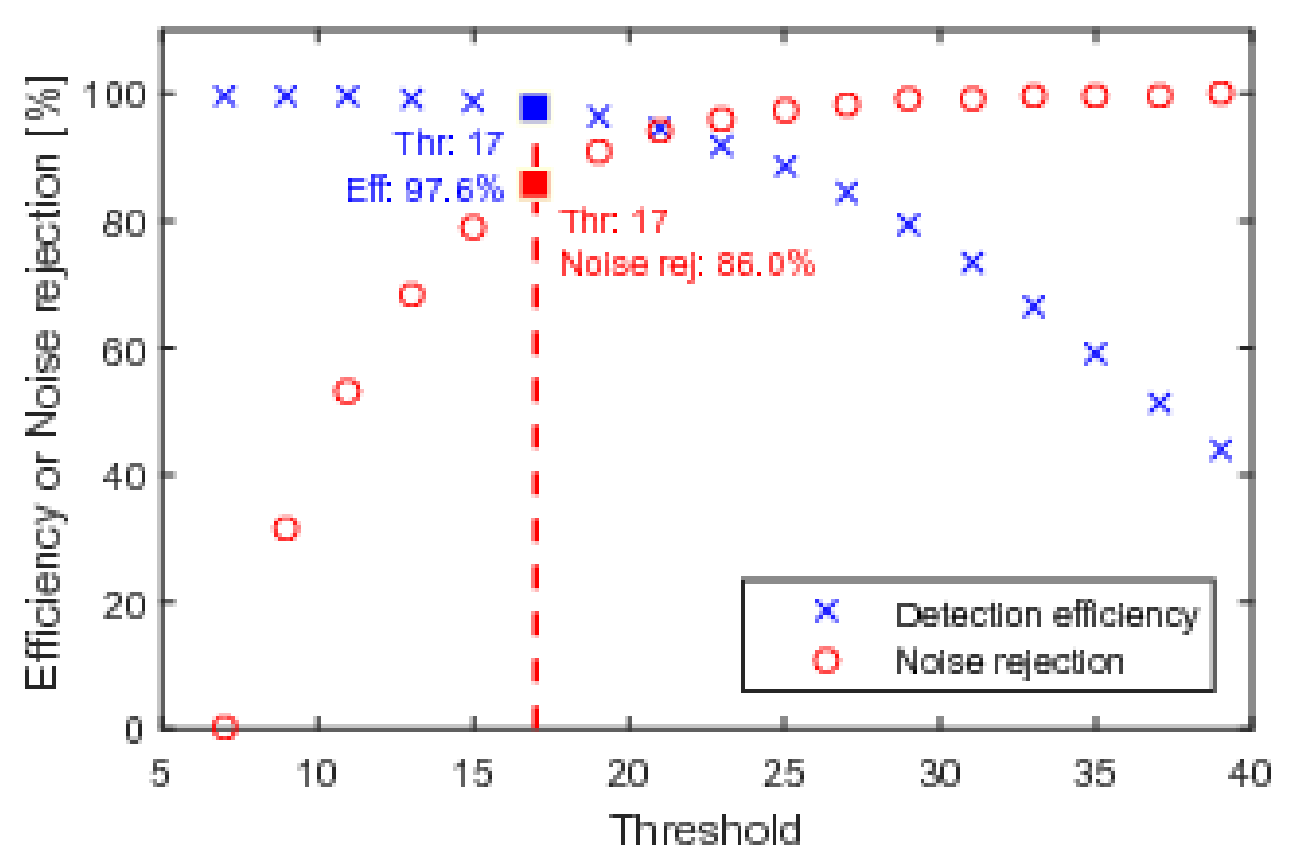

Efficiency and noise rejection

- Dark count rate:

- For 1000 Volt HV, the dark count rate was below $100 \mathrm{~Hz}$ per MaPMT.

- This is negligible compared to the expected hit rate

- Mean cross-talk rate : below $5 \%$ 


\section{SUMMARY}

- The LHCb RICH upgrade programme is well underway.

- $\quad$ For the RICH upgrade, the technical design review(TDR) completed in 2013.

- The engineering design reviews of various parts of the RICH system

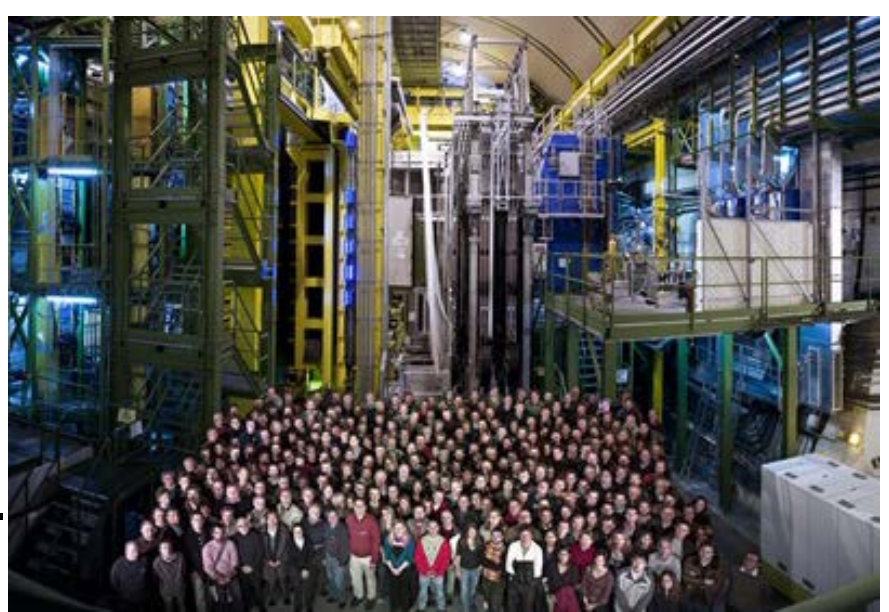
are in progress in 2016.

- It is expected to install the new detector during 2019-20

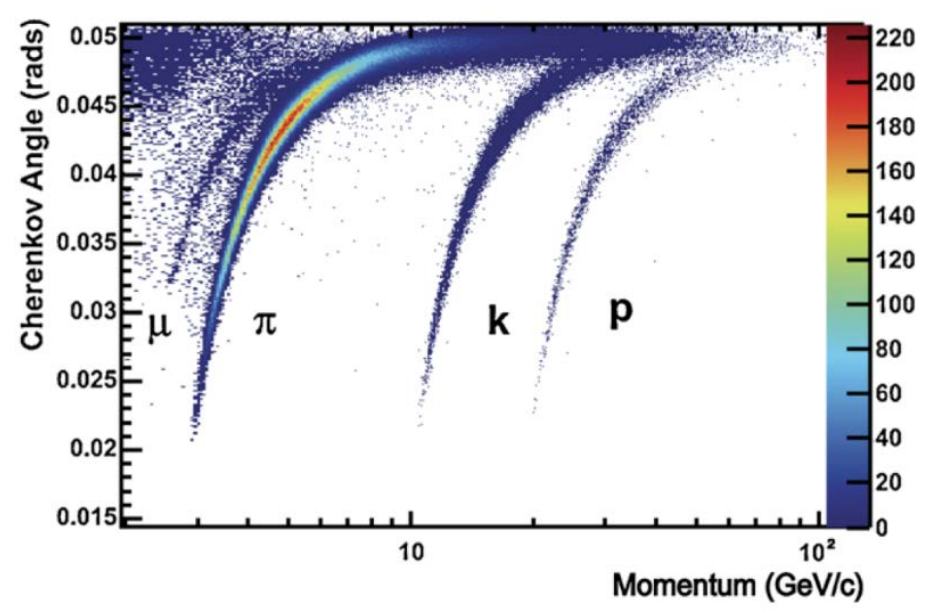

EPJC (2013) ,73:2431

From RICH1 gas radiator 


\section{BACKUP SLIDES}




\section{RICH2 Mechanics}

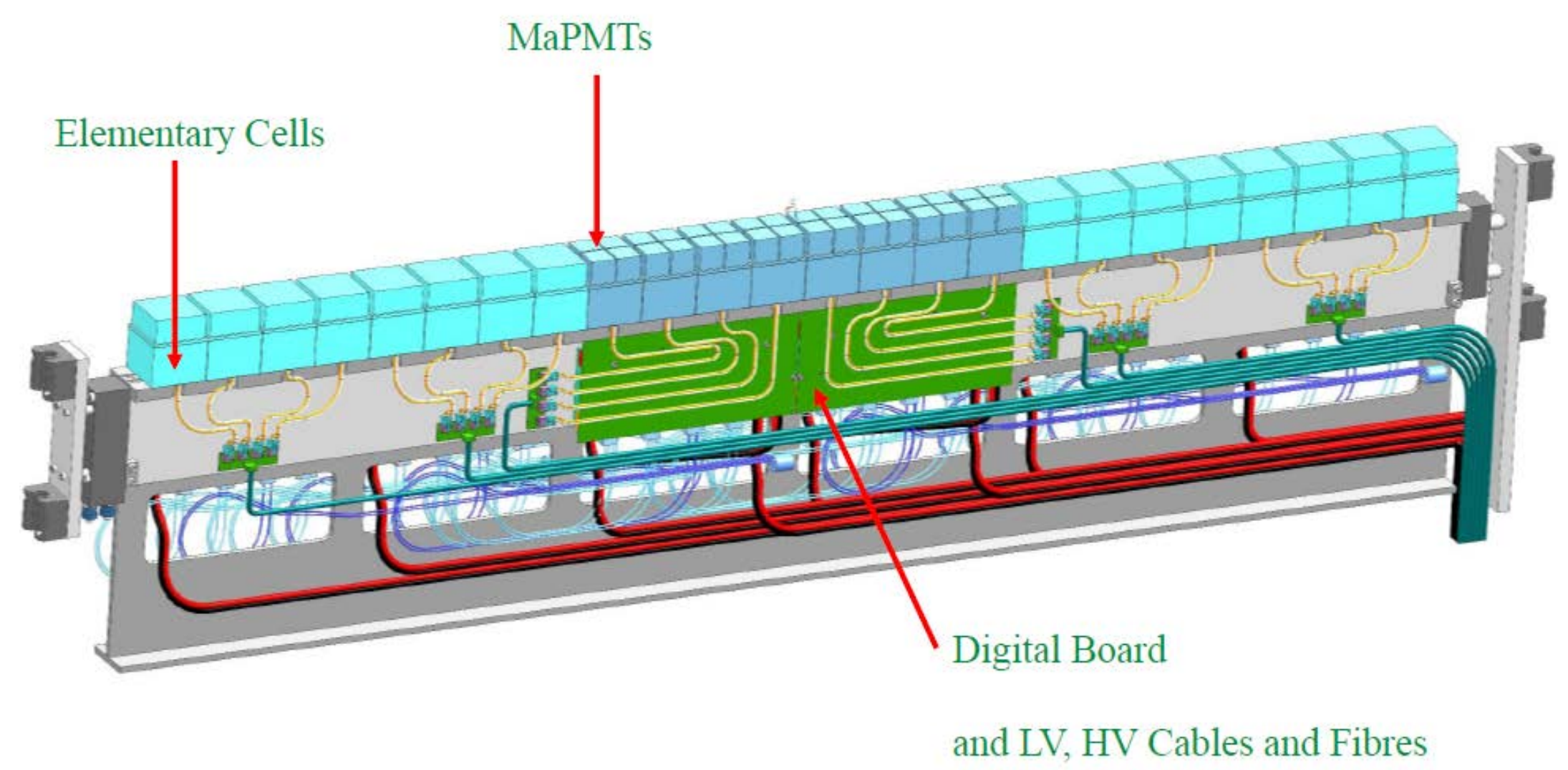

Photodetector Module (PDM) column in RICH2

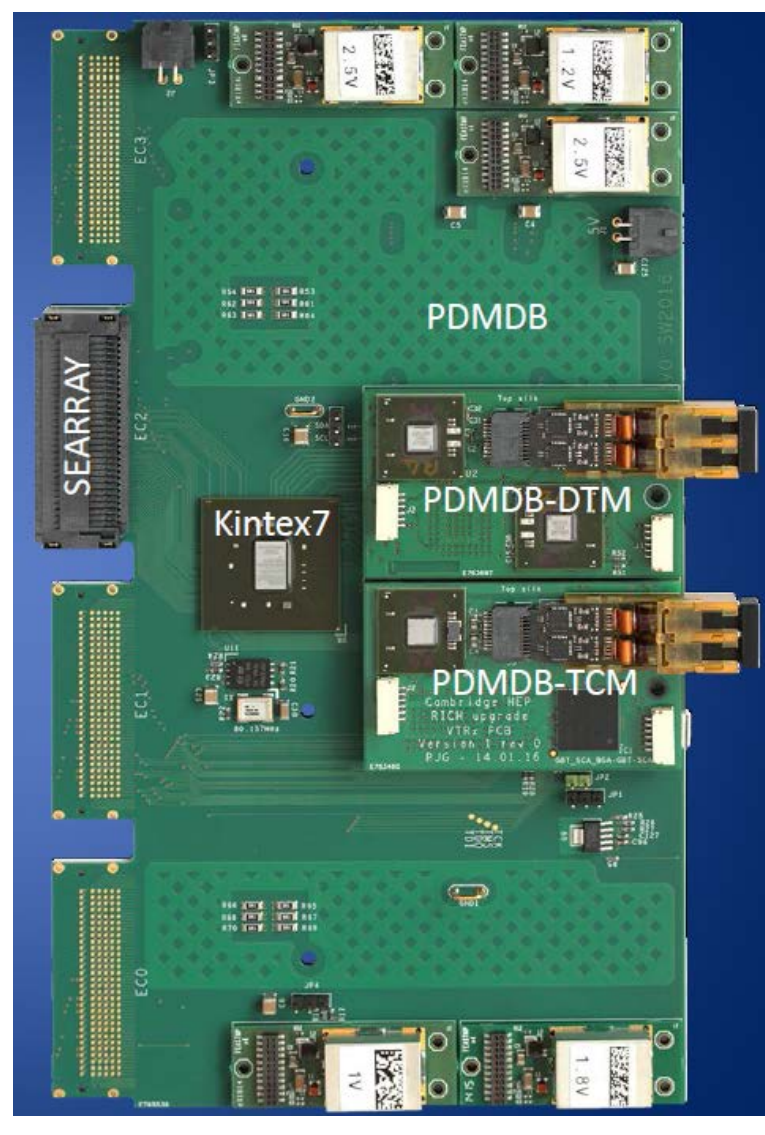

prototype of digital board 


\section{LHCb Trigger}

LHCb 2012 Trigger Diagram

$40 \mathrm{MHz}$ bunch crossing rate
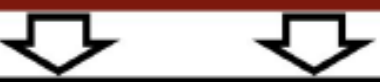

LO Hardware Trigger : 1 MHz readout, high $\mathrm{E}_{\mathrm{T}} / \mathrm{P}_{\mathrm{T}}$ signatures

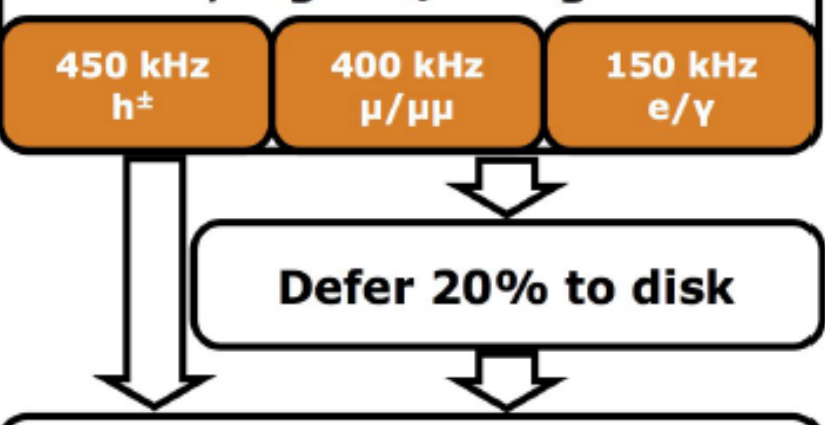

Software High Level Trigger 29000 Logical CPU cores

Offline reconstruction tuned to trigger time constraints

Mixture of exclusive and inclusive selection algorithms

Y

ए人

ए人

$5 \mathrm{kHz}(0.3 \mathrm{~GB} / \mathrm{s})$ to storage
LHCb 2015 Trigger Diagram

40 MHz bunch crossing rate
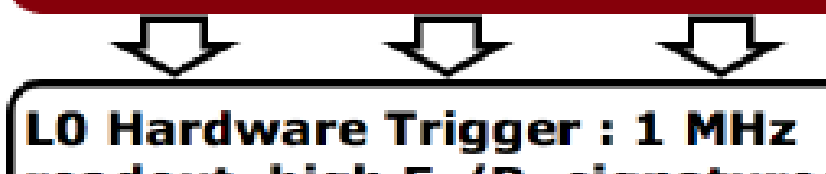
readout, high $\mathrm{E}_{\mathrm{T}} / \mathrm{P}_{\mathrm{T}}$ signatures

$450 \mathrm{kHz}$
$400 \mathrm{kHz}$

$h^{t}$

$\mu / \mu \mu$

e/v

$\sqrt{2}$

$\sqrt{2}$

Software High Level Trigger

Partial event reconstruction, select displaced tracks/vertices and dimuons 卢

Buffer events to disk, perform online detector calibration and alignment

$$
\text { Y }
$$

Full offline-like event selection, mixture of inclusive and exclusive triggers

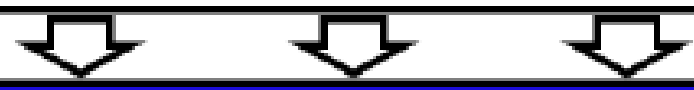

$12.5 \mathrm{kHz}$ Rate to storage

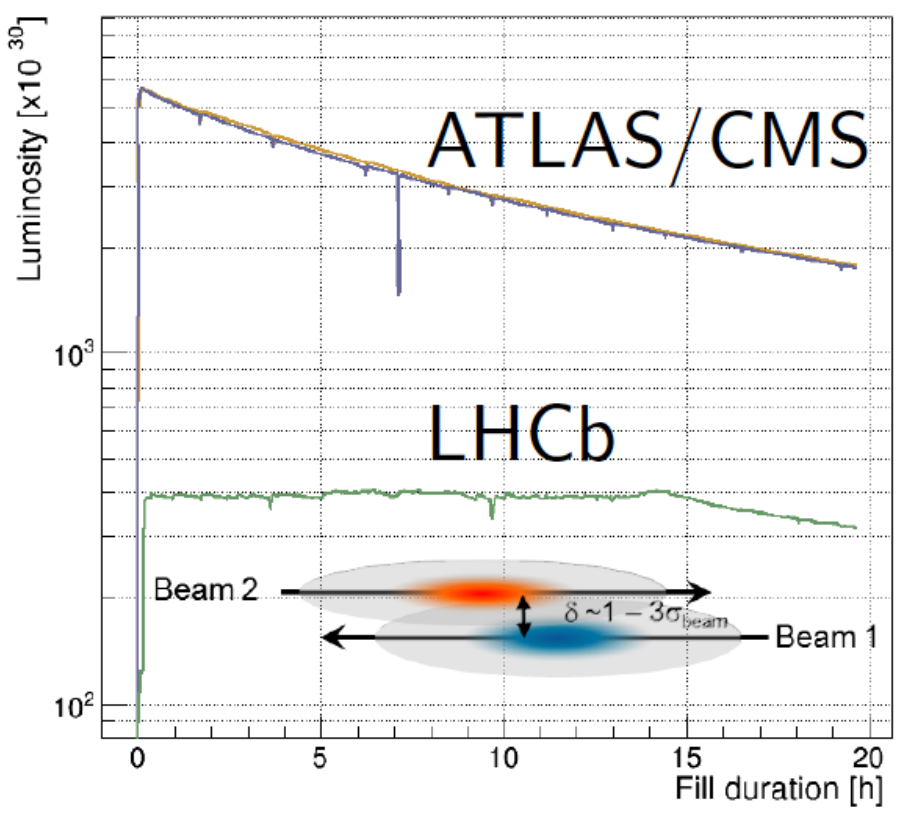




\section{PID vs momentum}

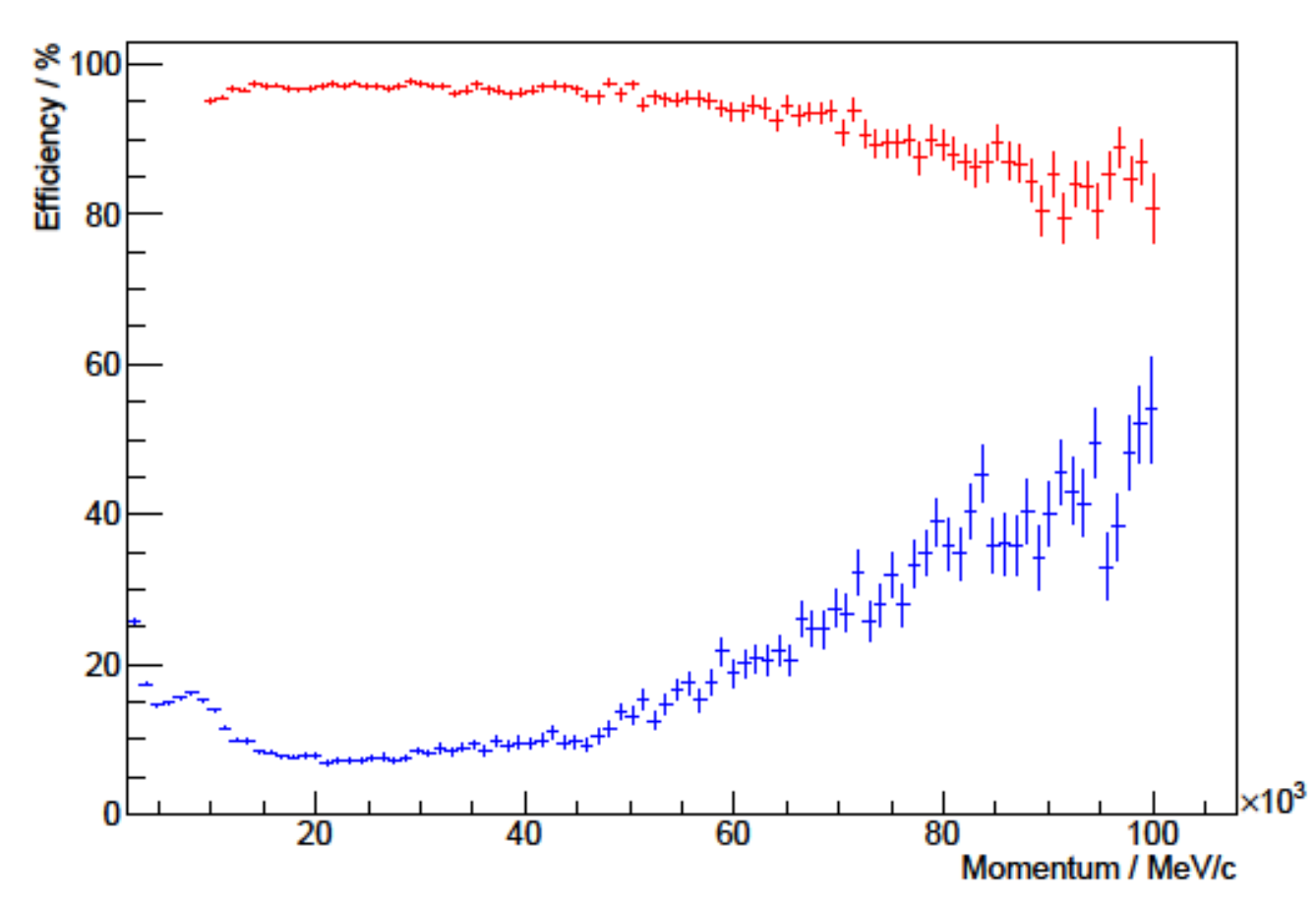

Current LHCb at Run 2 luminosity

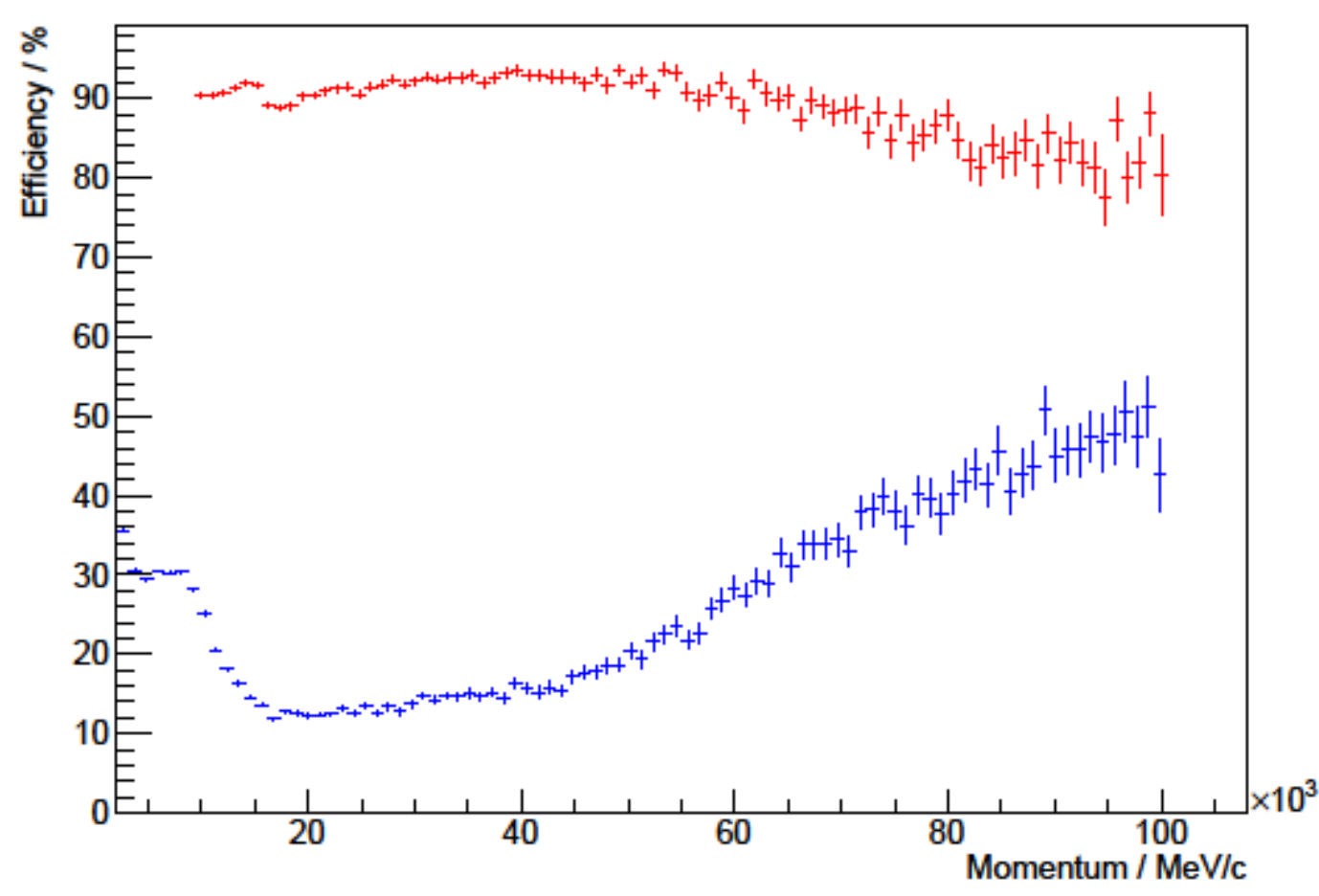

Current $\mathrm{LHCb}$ at Run 3 luminosity 
RICH1-2015 : Occupancy

XY Location of Rich1 Gas HPD hits on HPD detector Plane

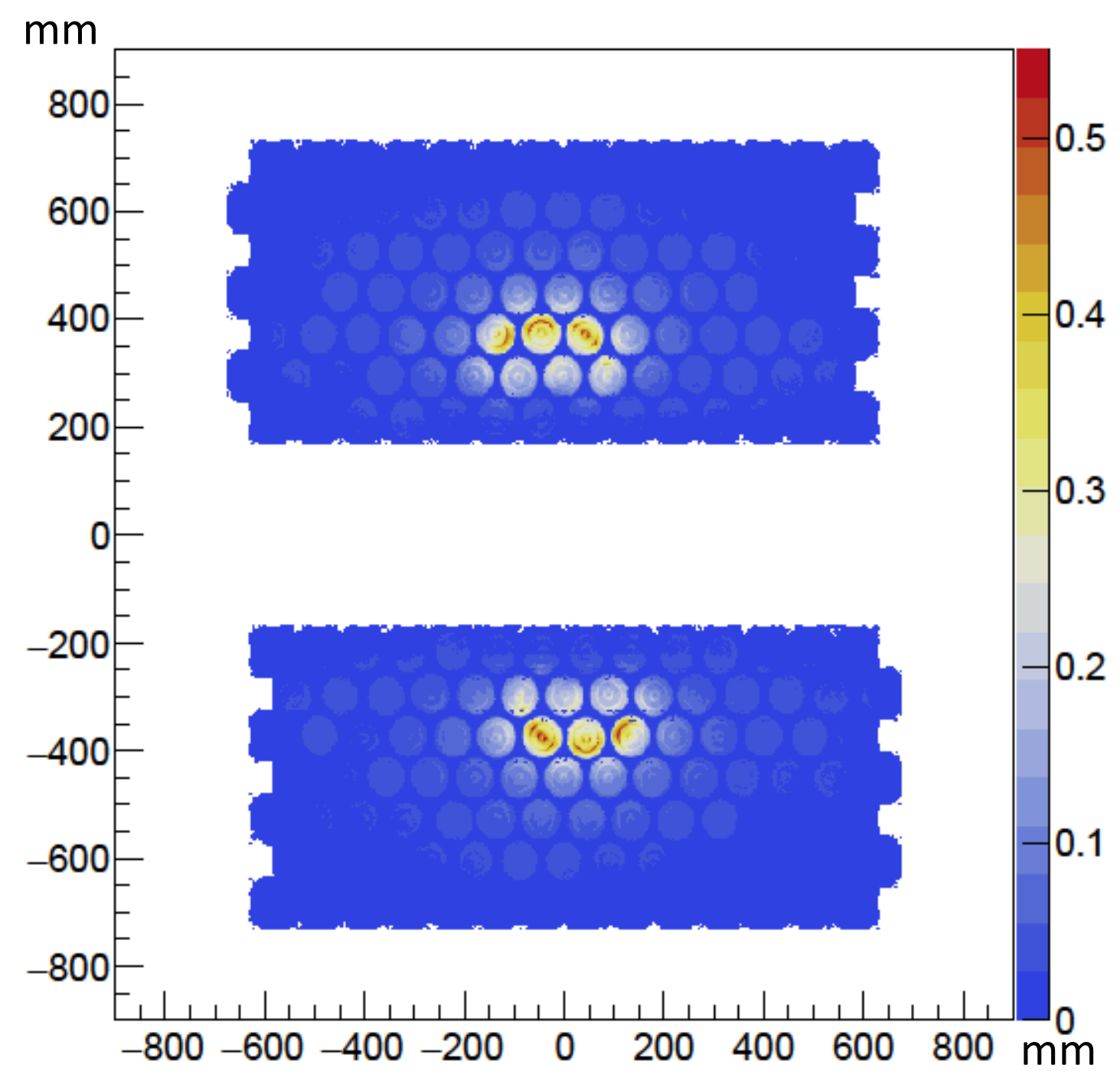

Occupancy at Run 3 luminosity 


\section{Nominal refractive index of the radiators}

C4F10 refractive index vs wavelength

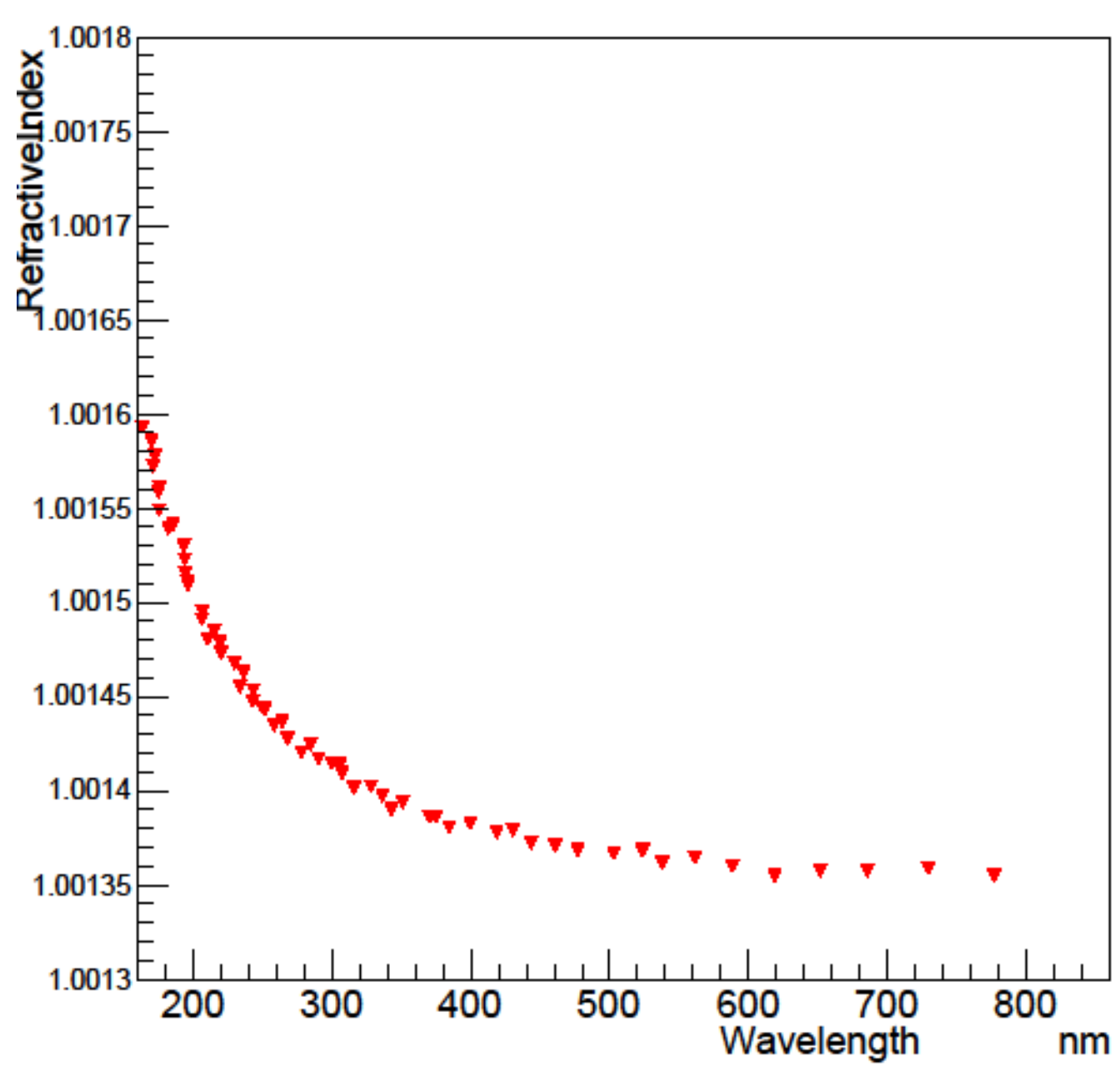

CF4 refractive index vs wavelength

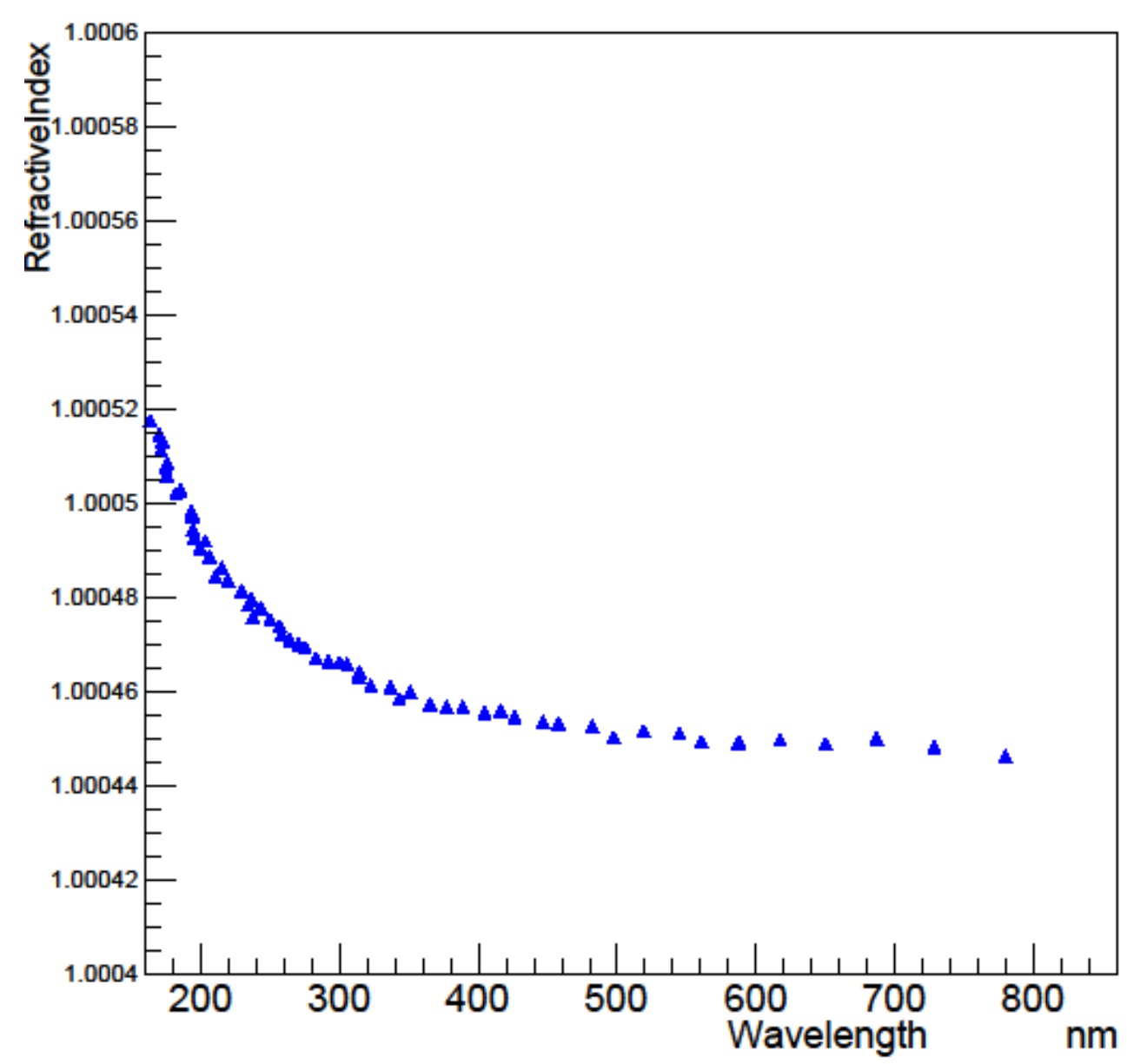

